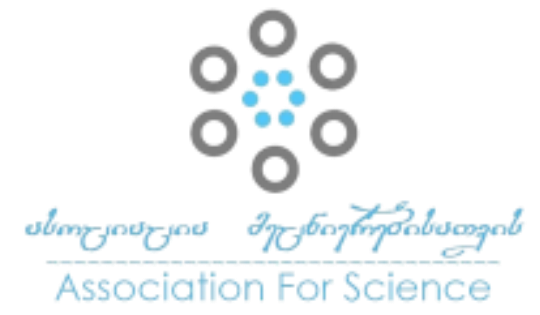

Georgian Scientists

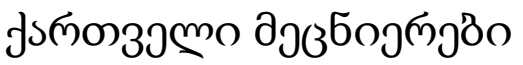

Vol. 4 Issue 1, 2022

https://doi.org/10.52340/gs.2022.04.01.05

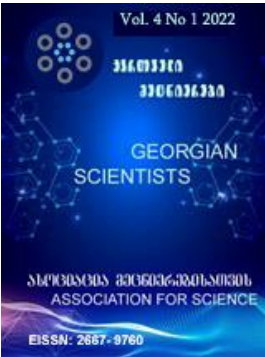

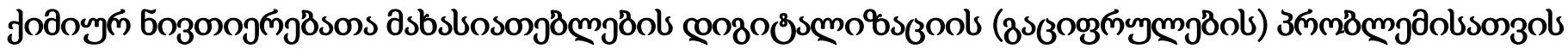

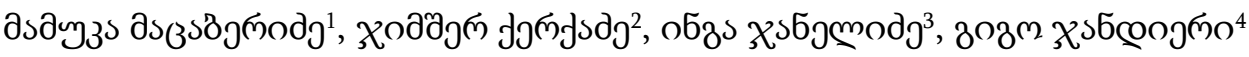

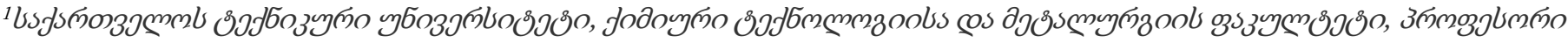

Orcid ID: https://orcid.org/0000-0002-3228-1447

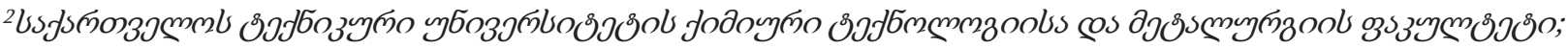

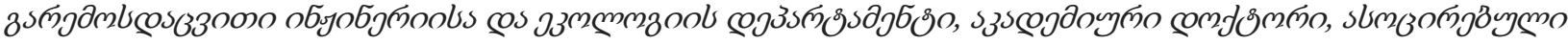

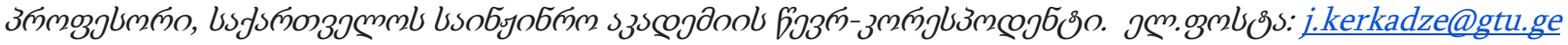

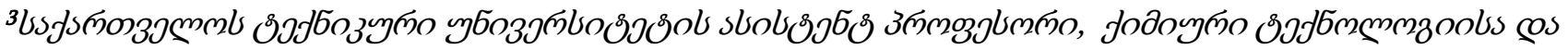

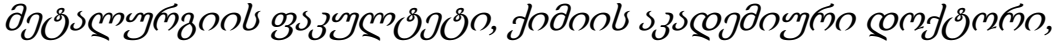

E-mail: i.janelidze@gtu.ge; Orcid ID: https://orcid.org/0000-0002-9961-7638

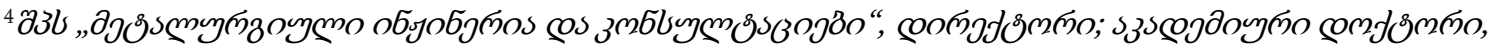

Jn-o3mbss: gigo.jandieri@gmail.com Orcid ID: https://orcid.org/0000-0003-2976-1201

\section{8oygy}

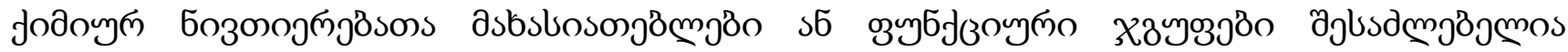

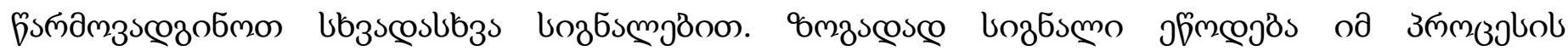

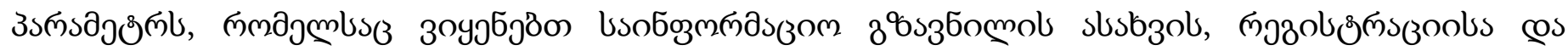

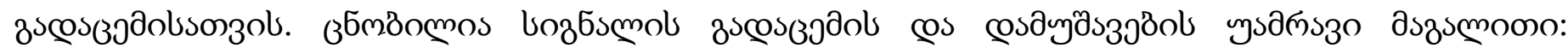

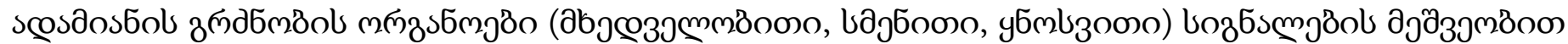

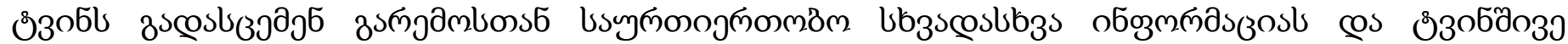

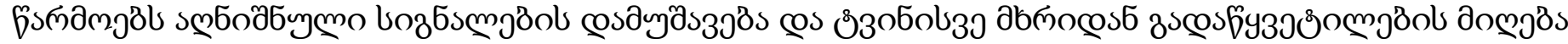

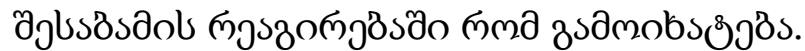

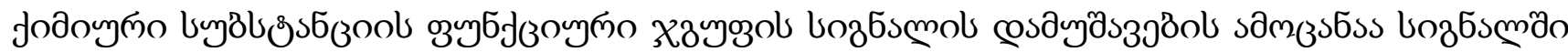

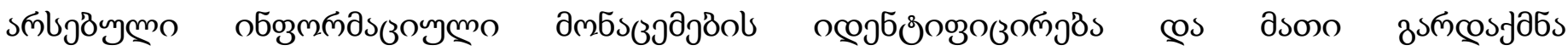

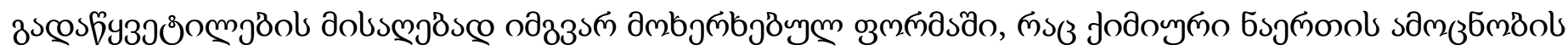

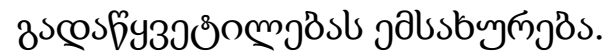




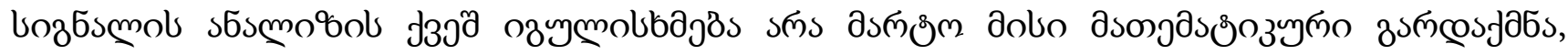

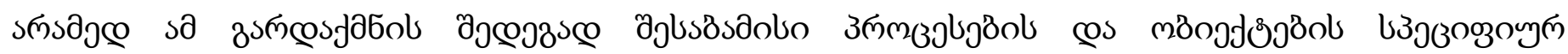

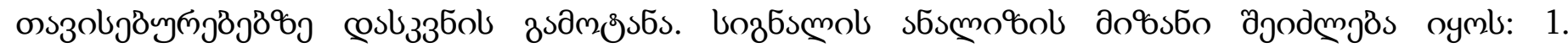

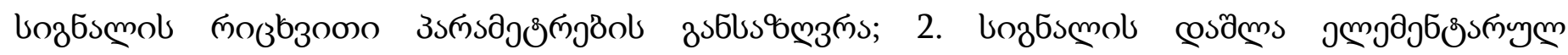

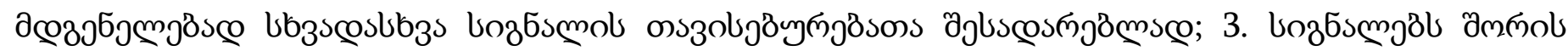

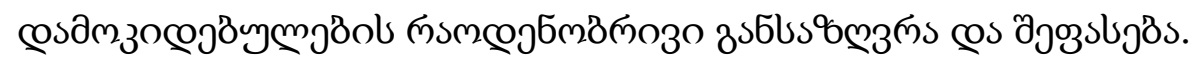

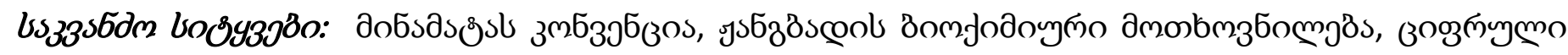

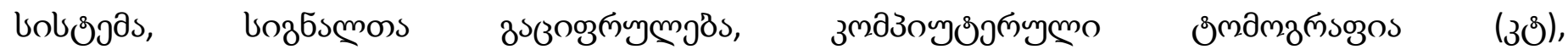

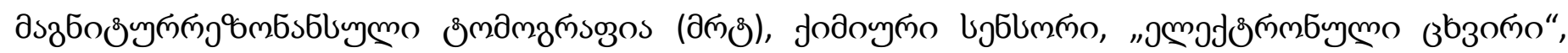

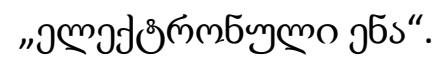

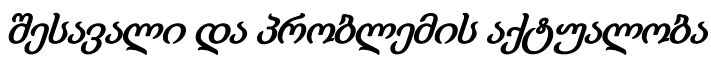

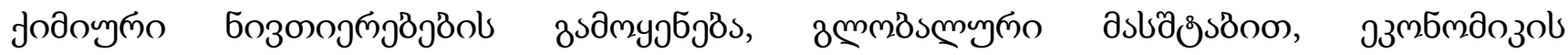

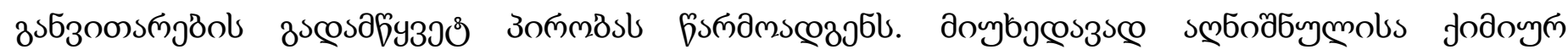

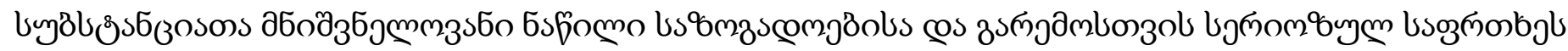
бьпдмзмаэбl.

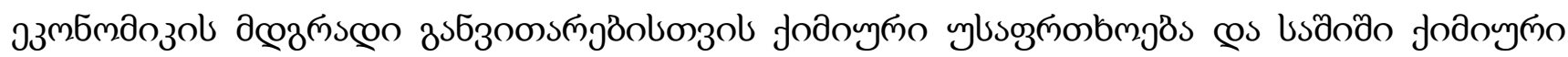

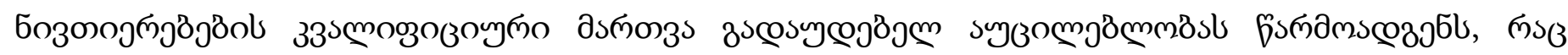

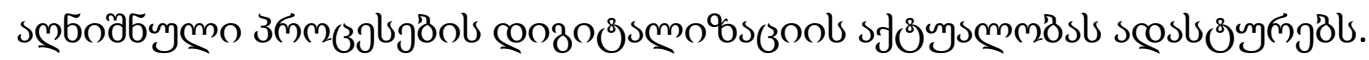

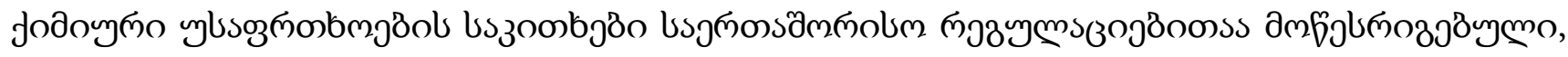

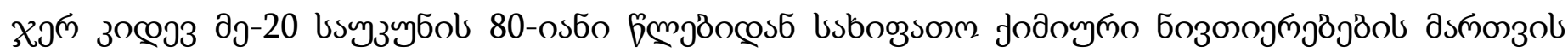

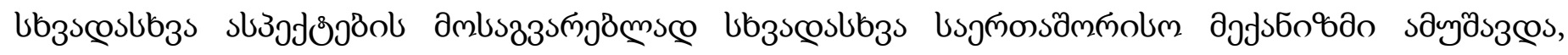

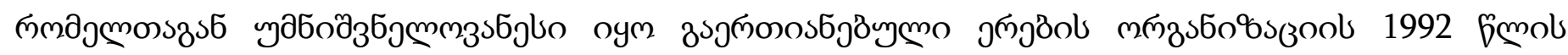

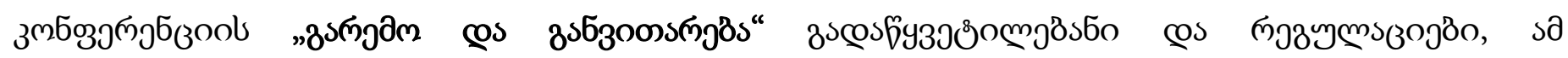

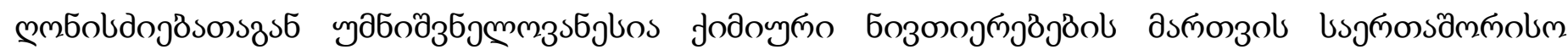

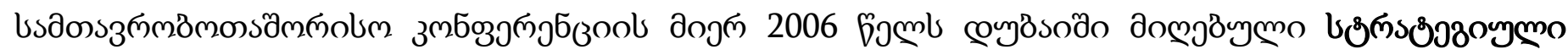

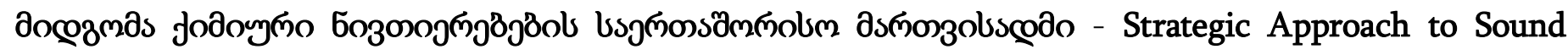

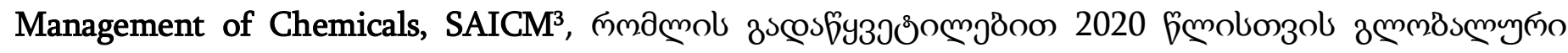

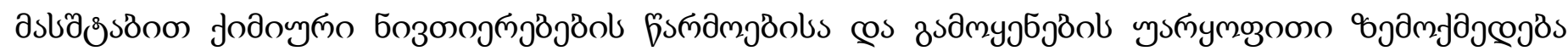

\footnotetext{
${ }^{3}$ Adopted by the First International Conference on Chemicals Management (ICCM1) on 6 February 2006 in Dubai, the Strategic Approach to International Chemicals Management (SAICM) is a policy framework to promote chemical safety around the world. SAICM was developed by a multi-stakeholder and multi-sectoral Preparatory Committee and supports the achievement of the 2020 goal agreed at the 2002 Johannesburg World Summit on Sustainable Development. SAICM overall objective is the achievement of the sound management of chemicals throughout their life cycle so that by the year 2020, chemicals are produced and used in ways that minimize significant adverse impacts on the environment and human health. http://www.saicm.org/About/SAICMOverview/tabid/5522/language/en-US/Default.aspx
} 


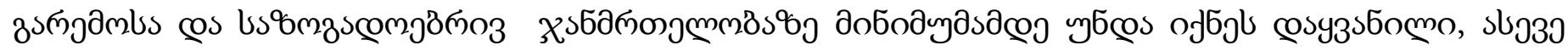

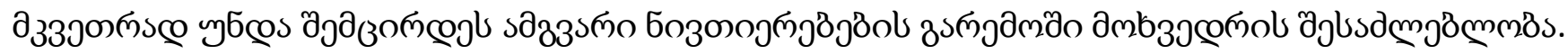

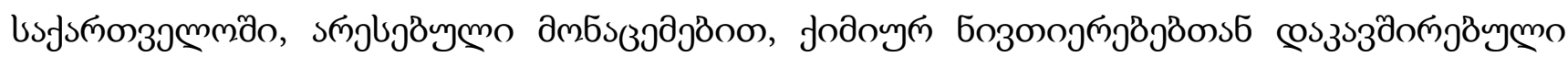

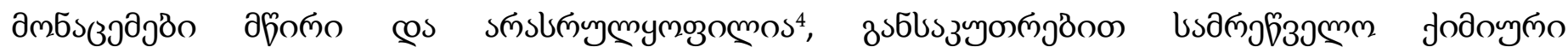

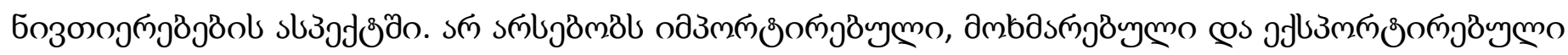

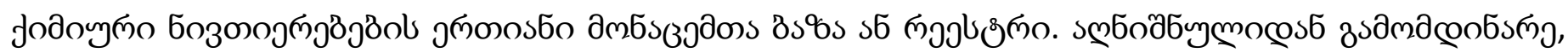

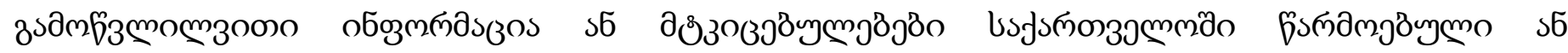

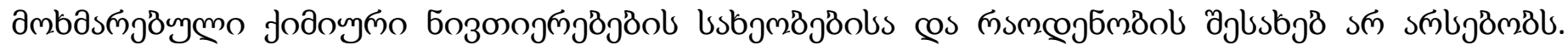

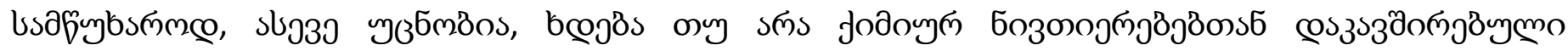

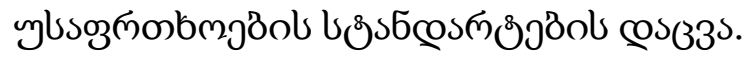

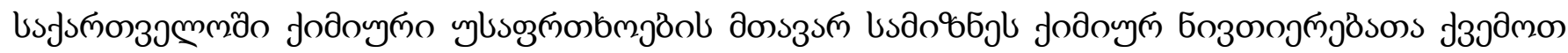

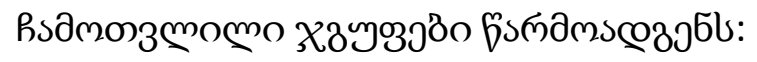

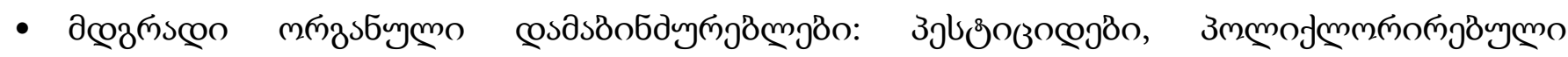

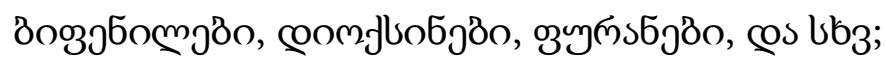

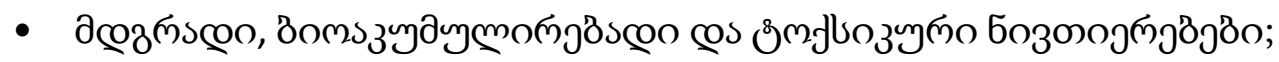

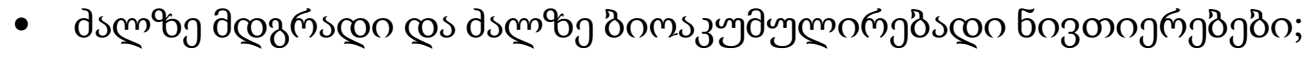

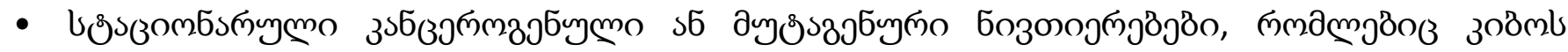

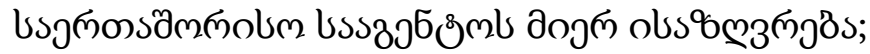

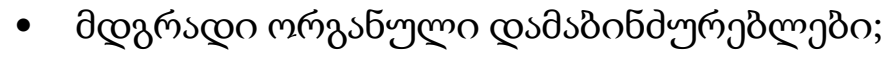

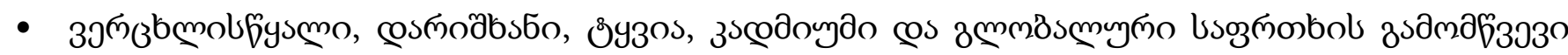
ubzs lugalsos

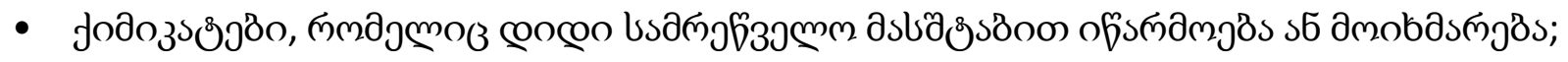

- usluyjjöo;

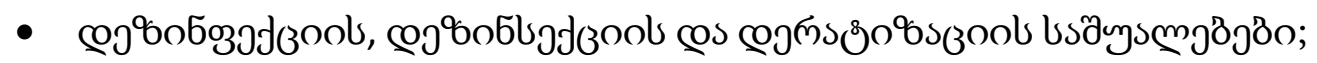

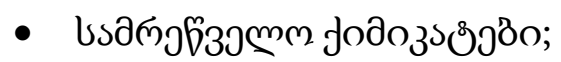

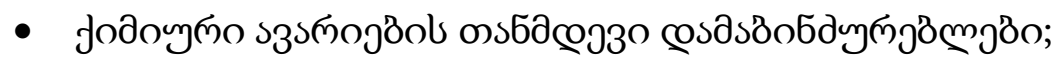

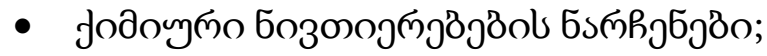

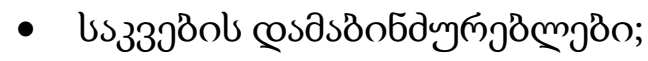

- Eoscoszol cos fymol cosas

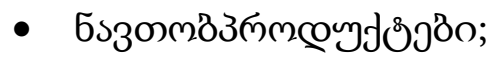

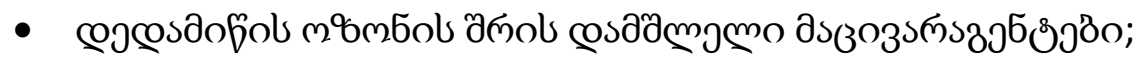

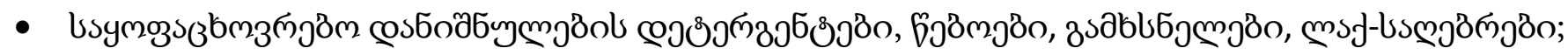

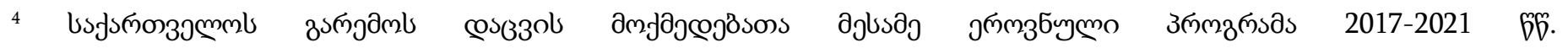
http://moe.gov.ge/res/images/file-manager/strategiuli-dokumentebi/strategiebi-gegmebi/2017-2021.pdf 


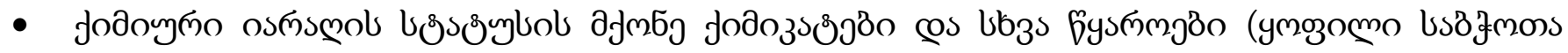

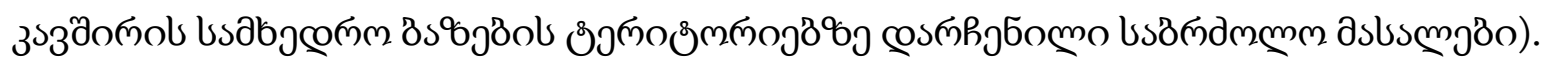

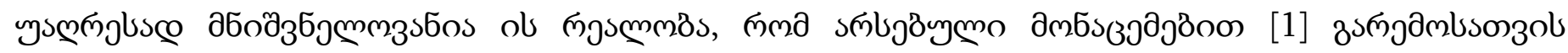

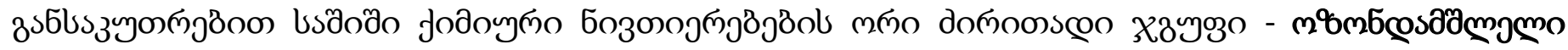

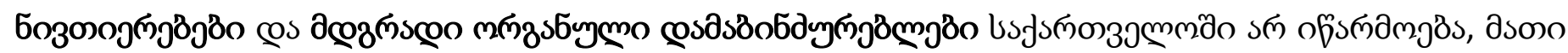

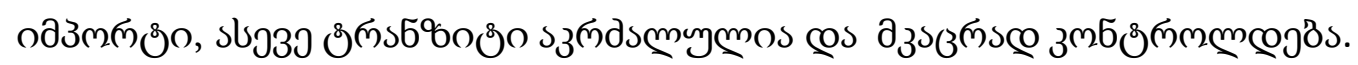

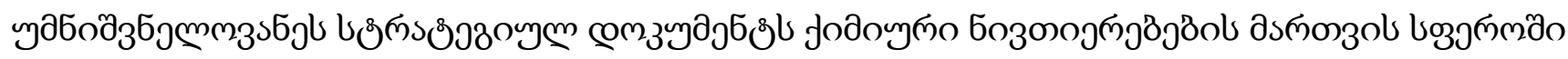

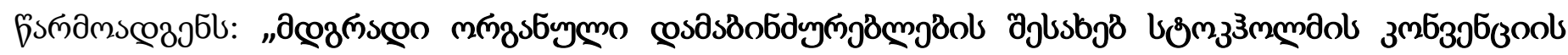

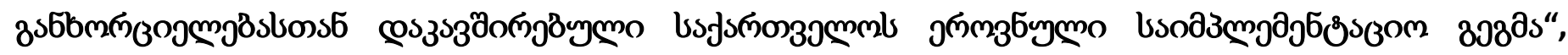

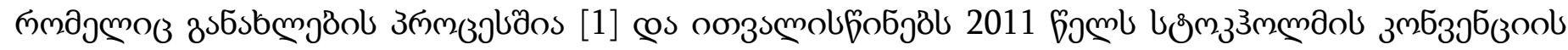

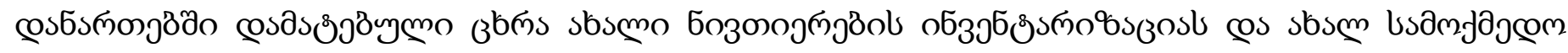

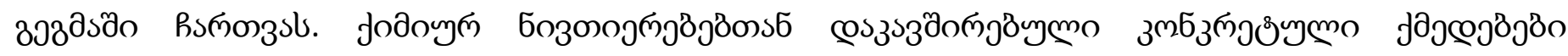

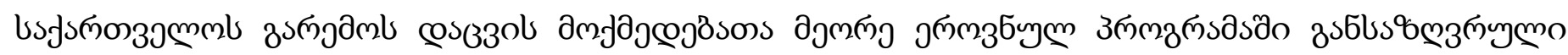
smss.

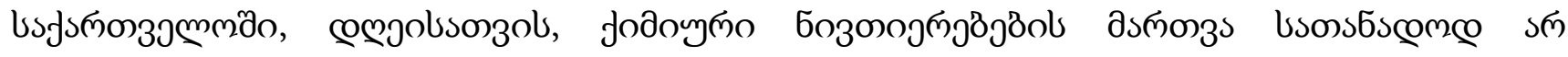

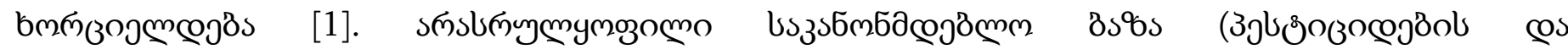

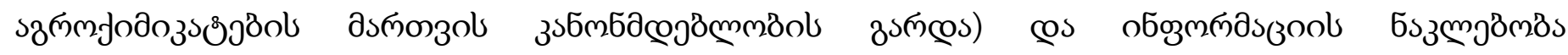

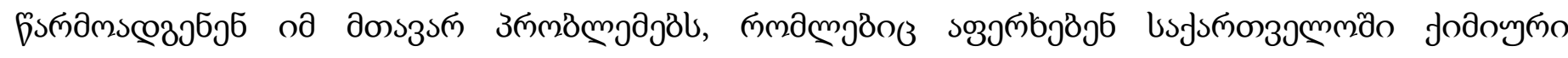

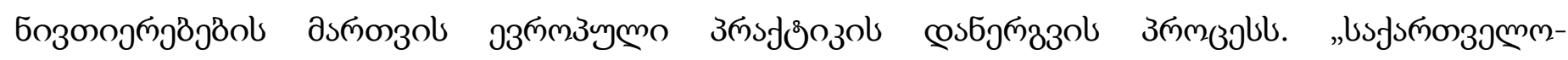

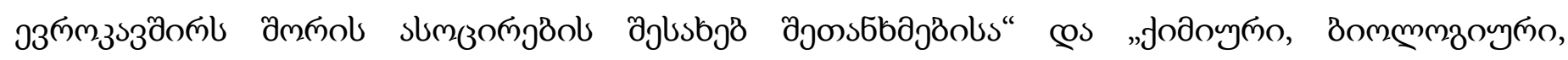

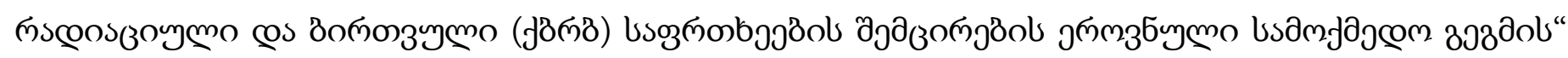

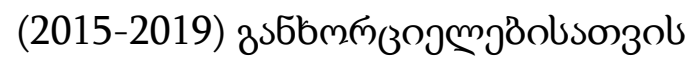

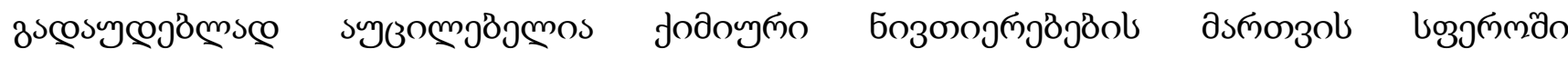

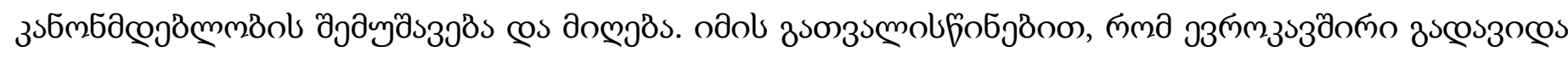

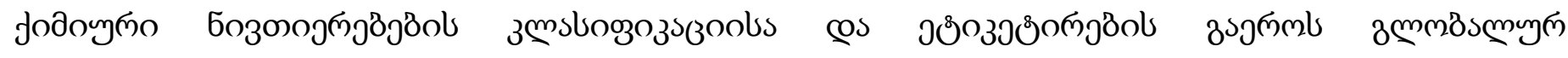

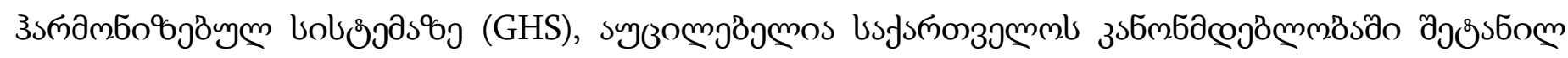

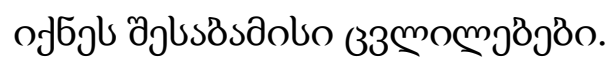

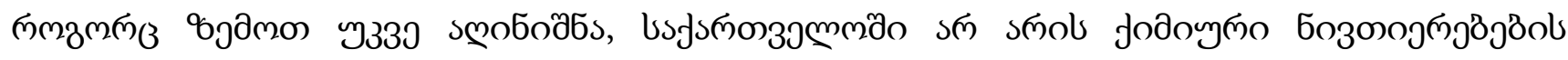

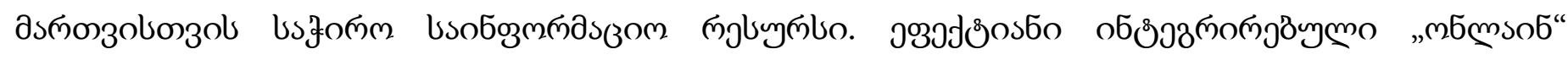

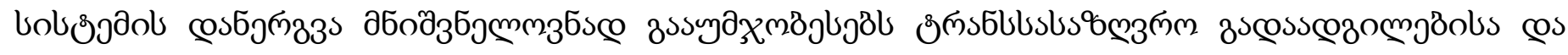

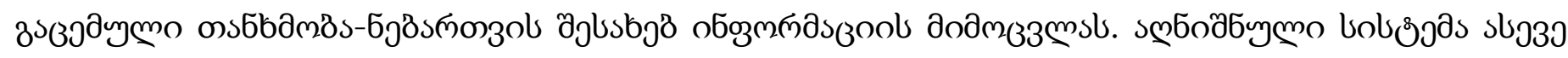

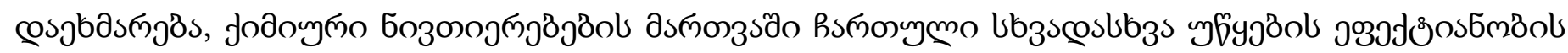
sasmegissl.

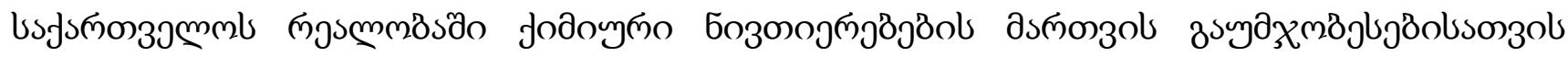

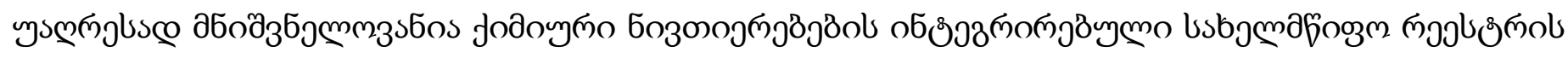

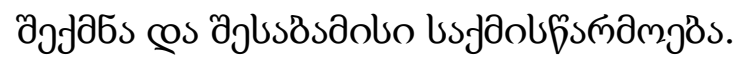




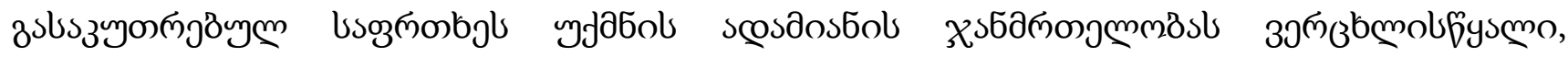

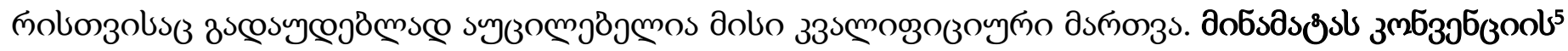

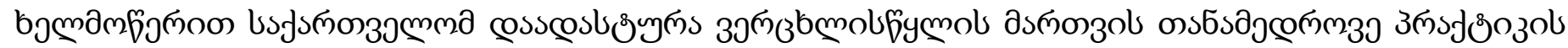

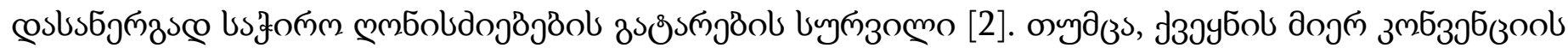

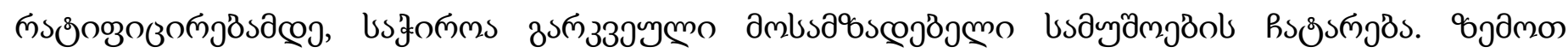

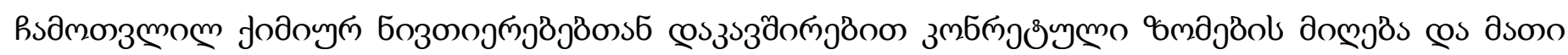

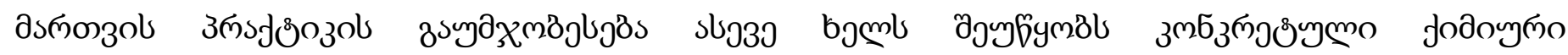

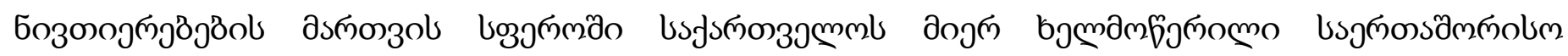

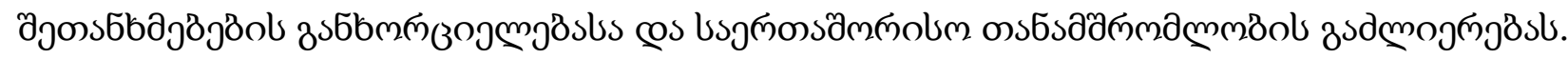

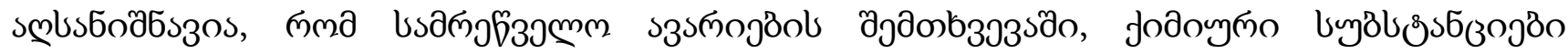

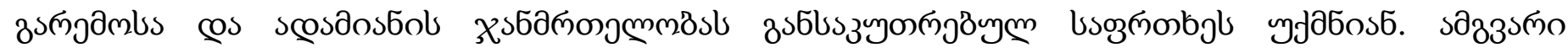

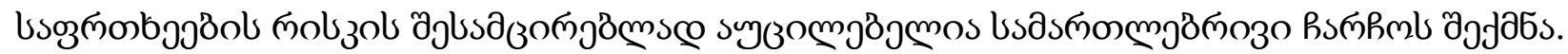

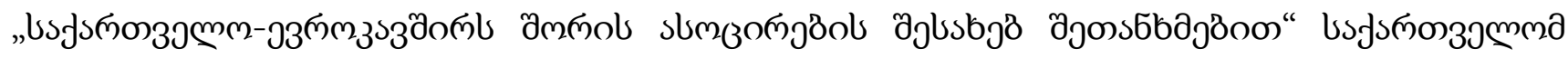

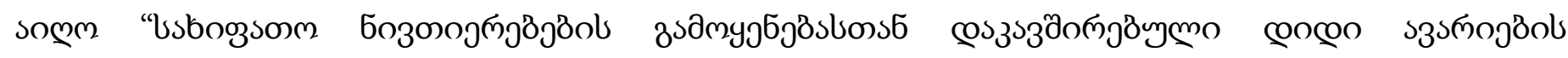

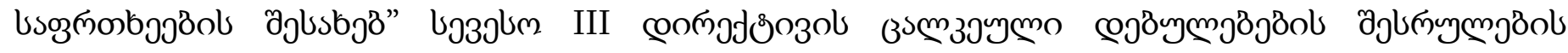

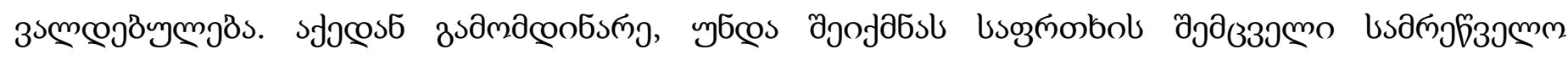

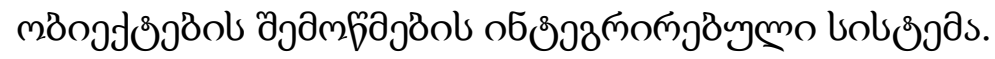

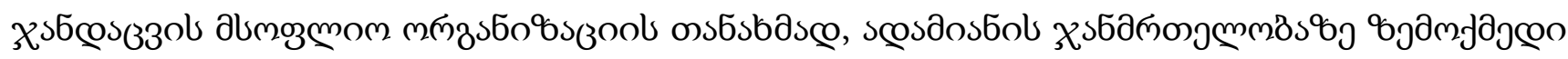

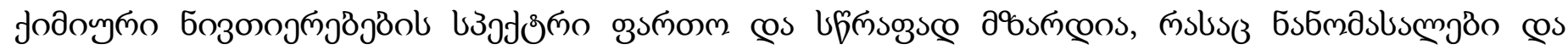

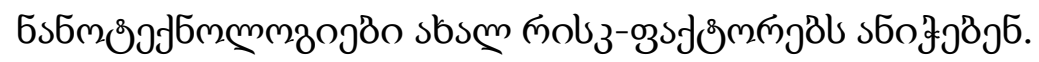

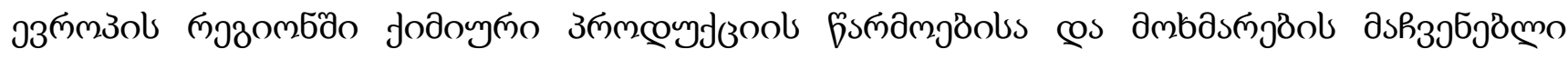

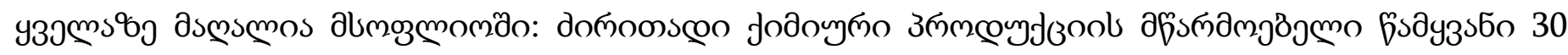

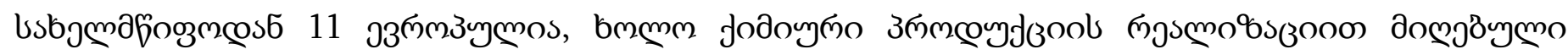

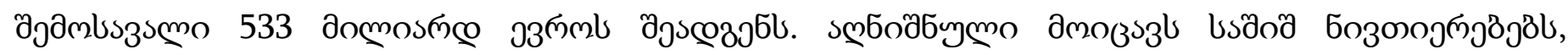

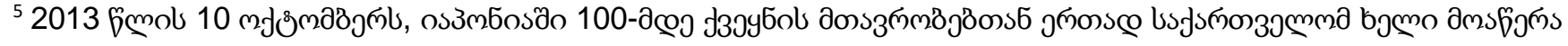

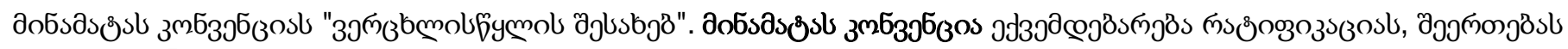

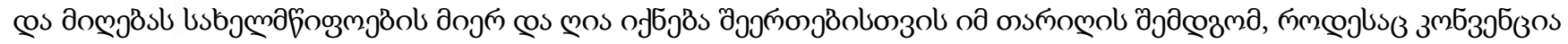

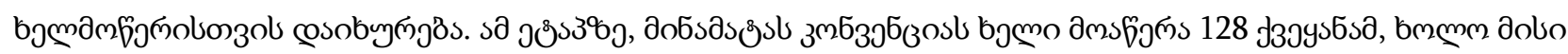

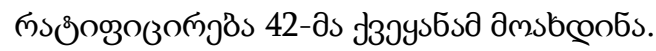

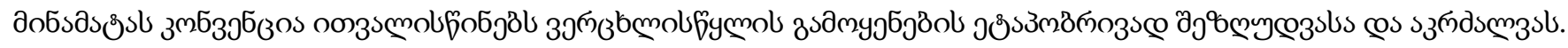

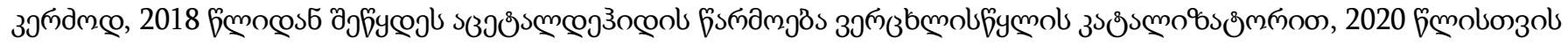

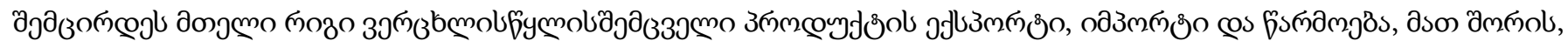

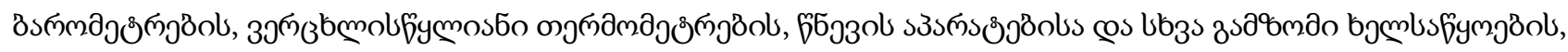

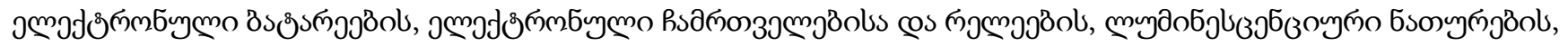
змб

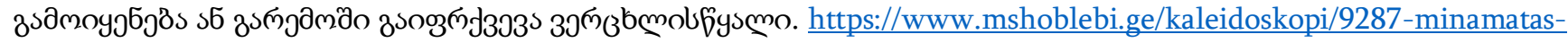
incidenti-da-minamatas-konvencia-qverckhlistsylis-shesakhebq.html
} 


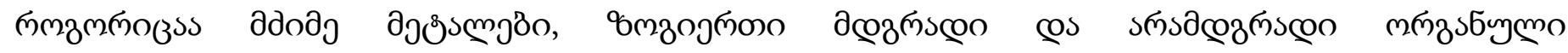

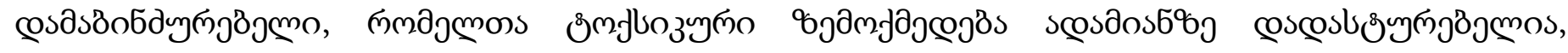

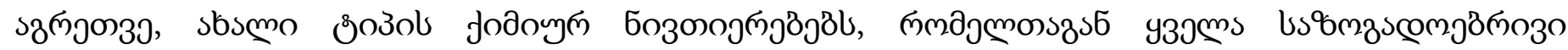

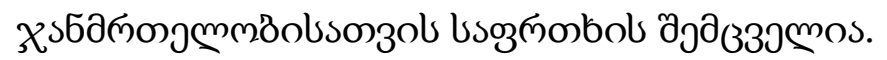

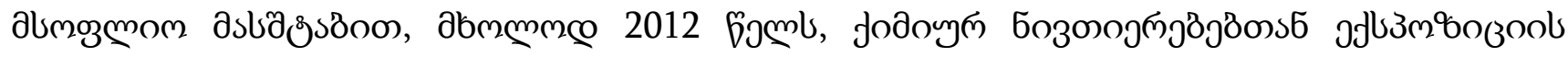

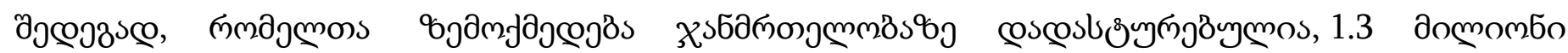
бssc๓

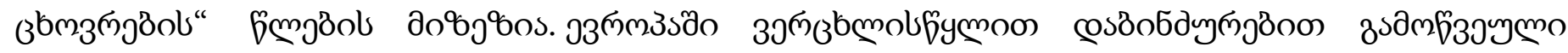

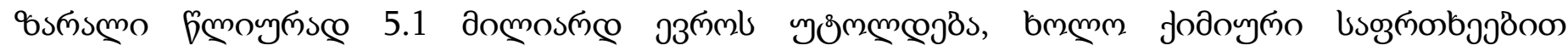

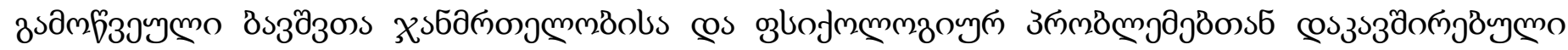

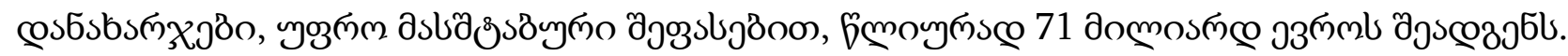

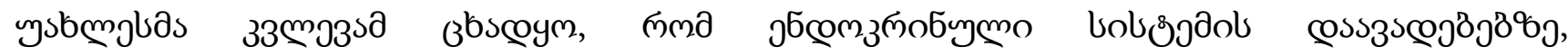

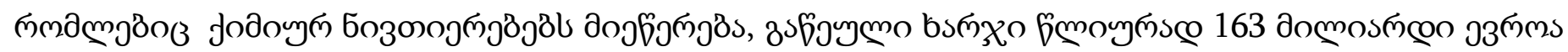
[3-4].

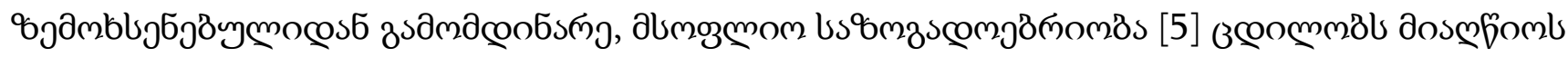

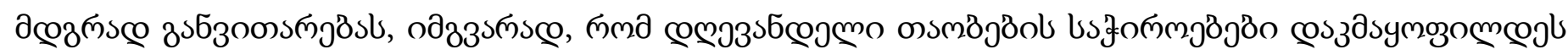

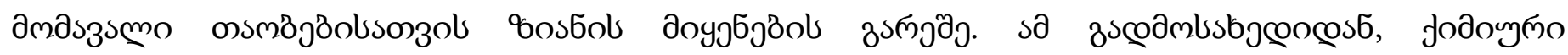

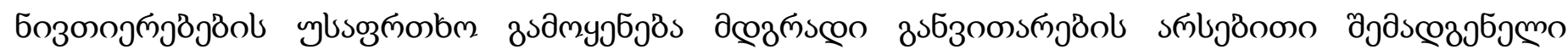
бsfomos.

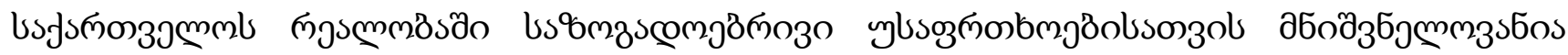

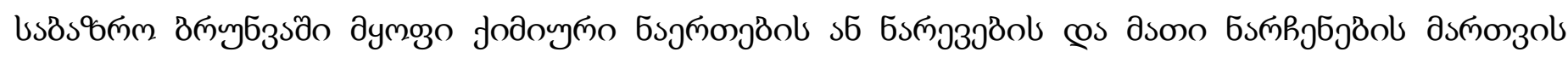

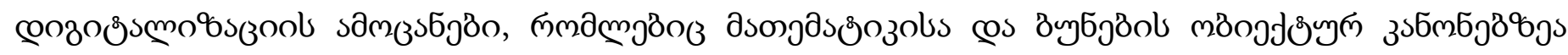

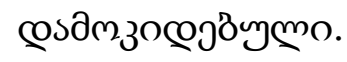

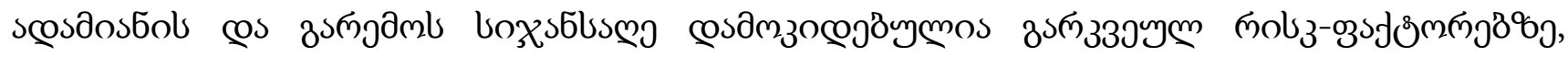

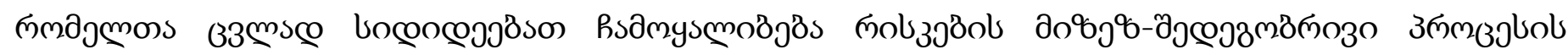

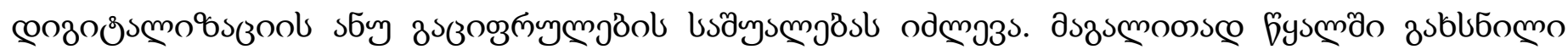

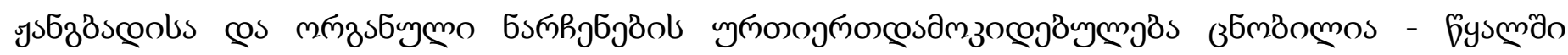

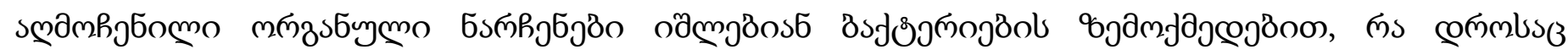

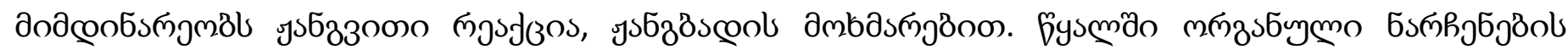

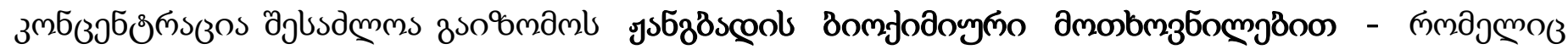

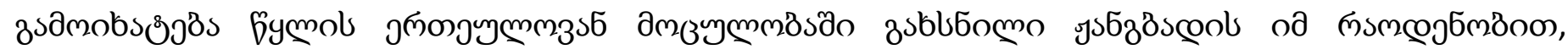

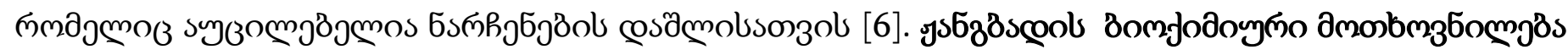

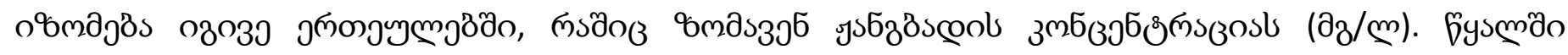

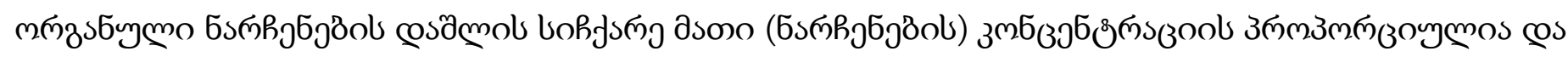




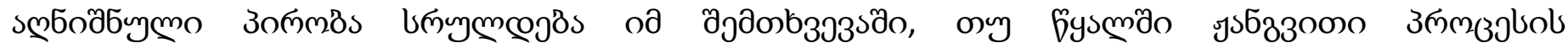

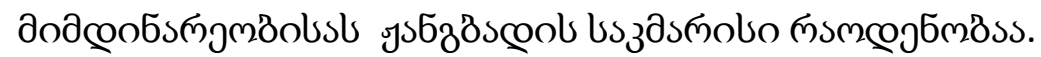

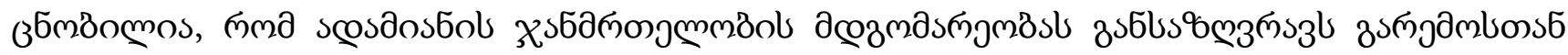

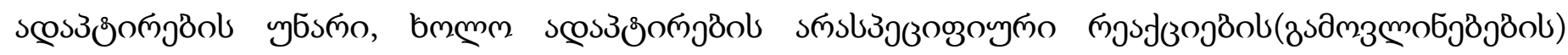

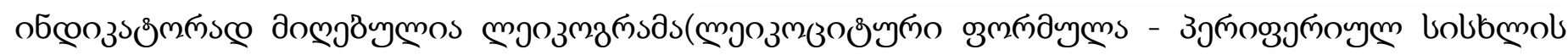

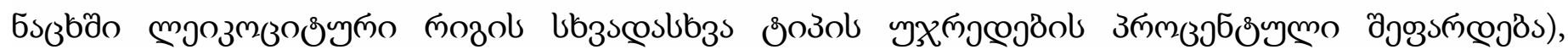

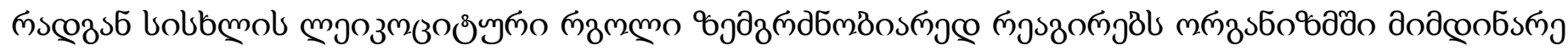

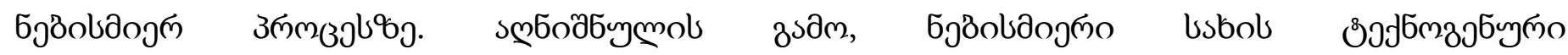

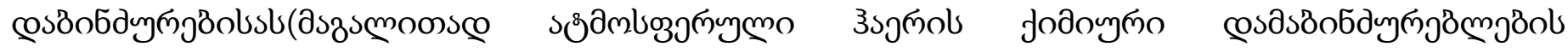

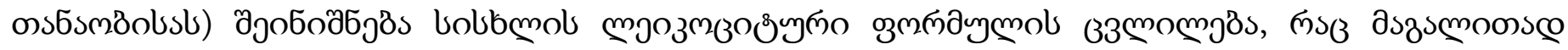

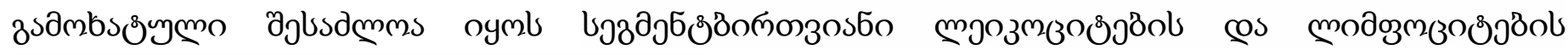

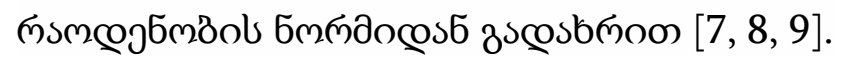

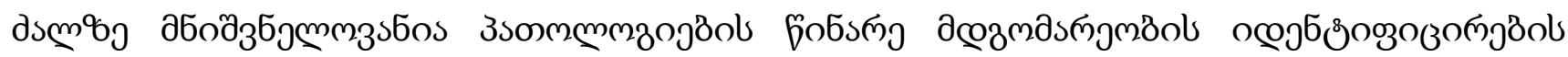

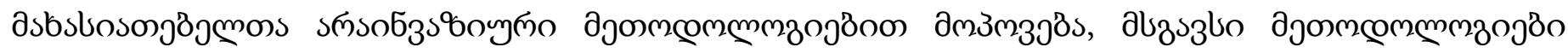

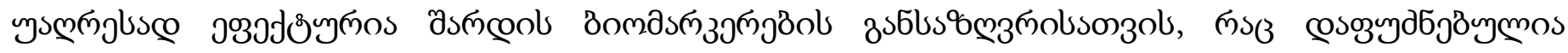

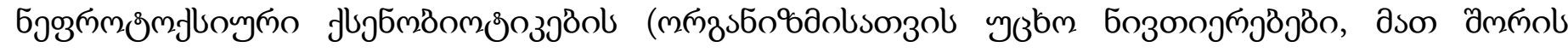

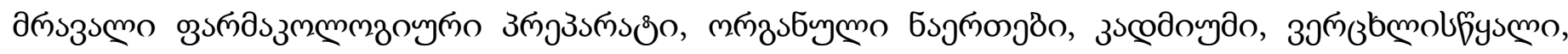

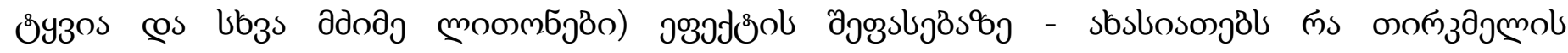

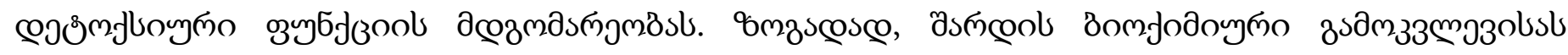

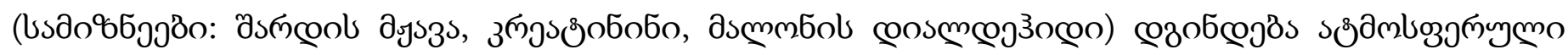

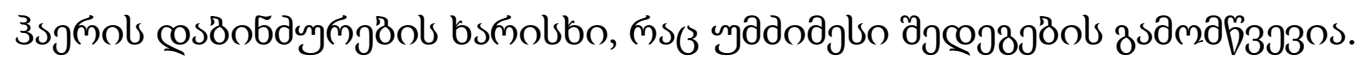

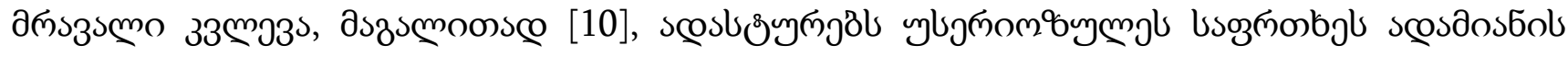

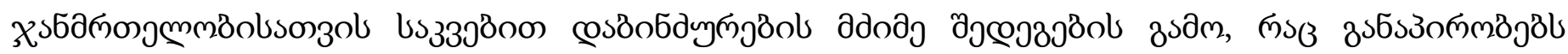

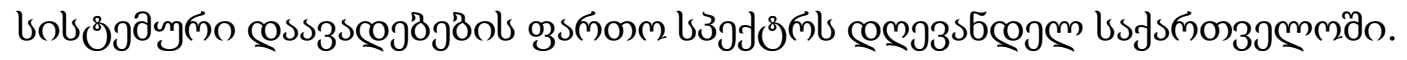

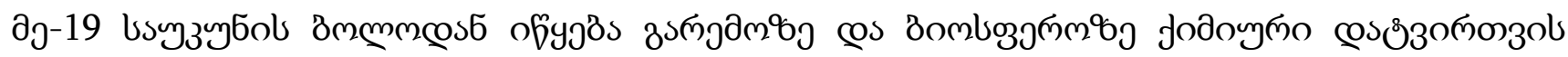

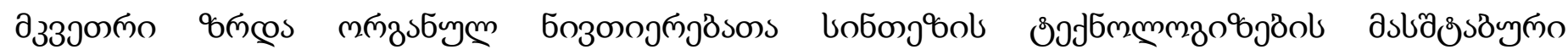

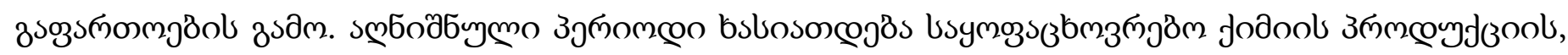

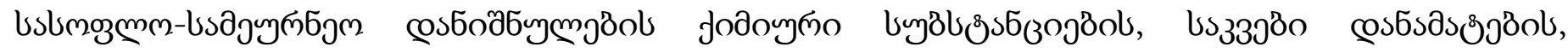

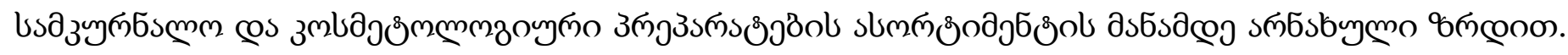

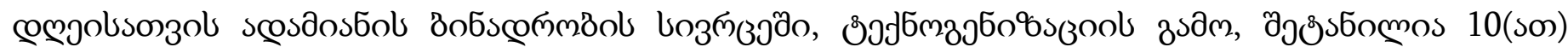

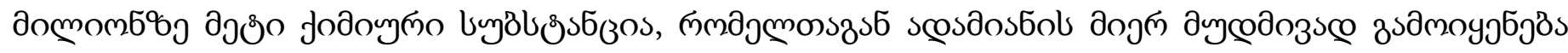

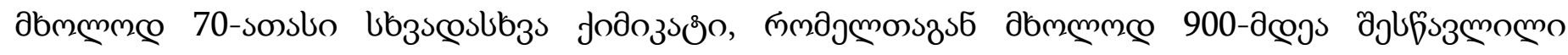

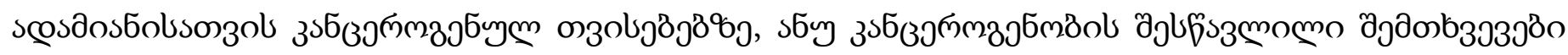

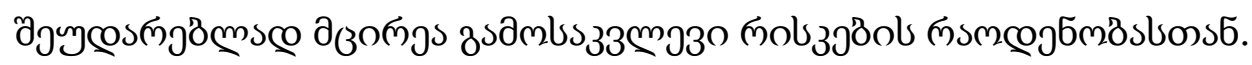

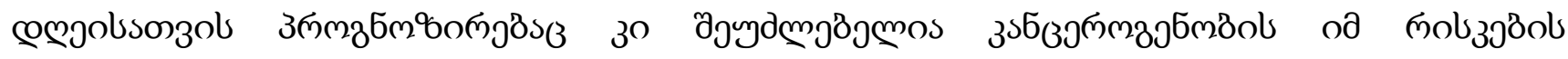

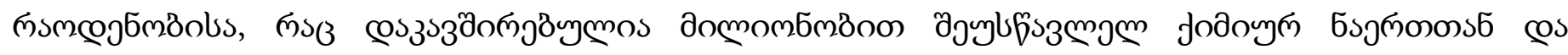




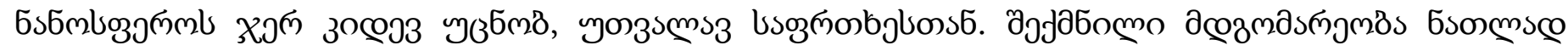

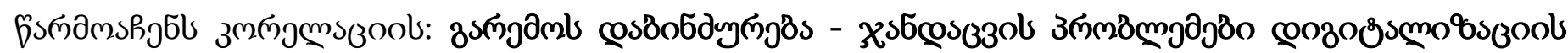

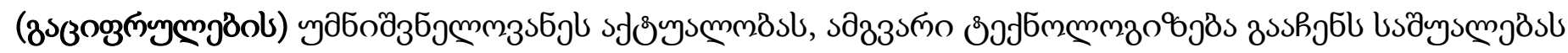

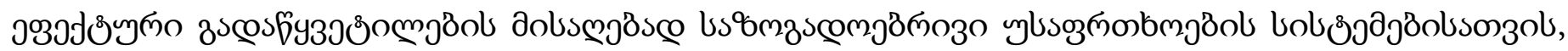

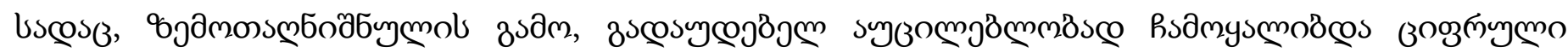

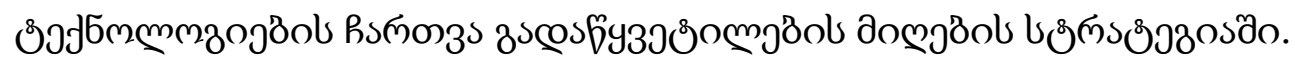

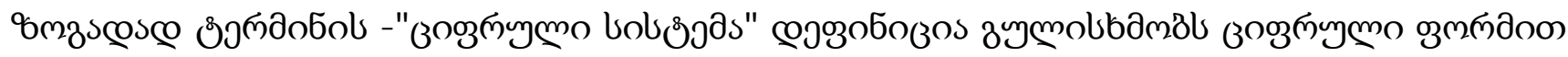

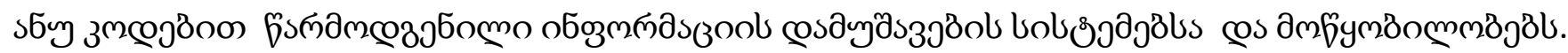

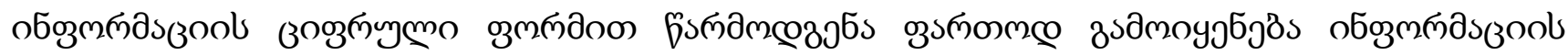

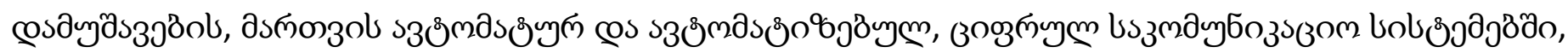

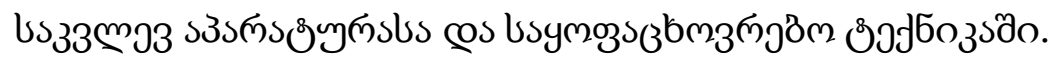

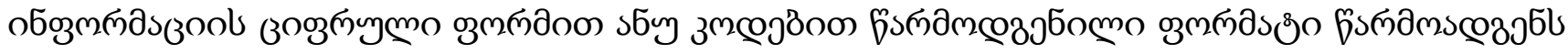

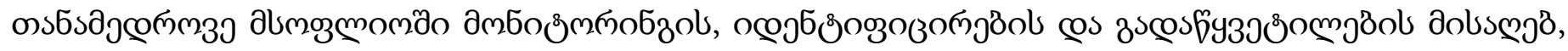

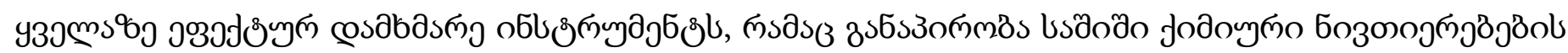

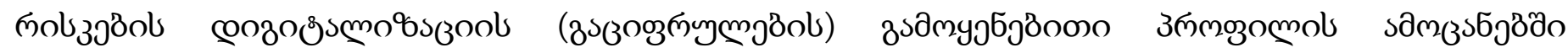

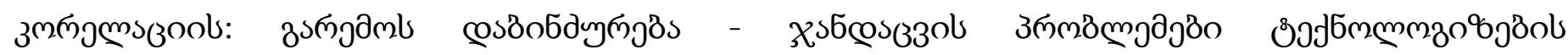

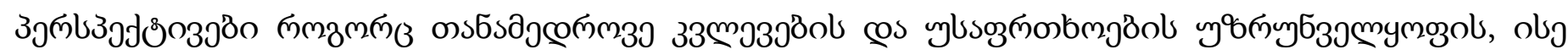

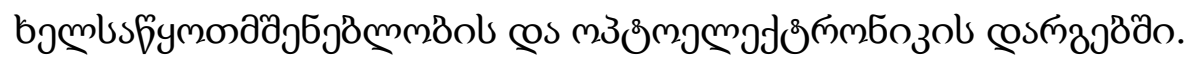

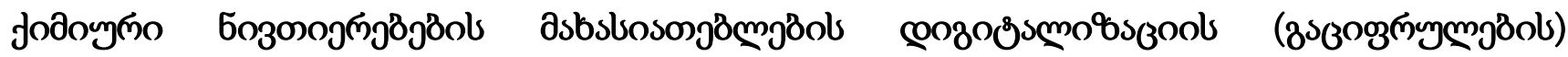

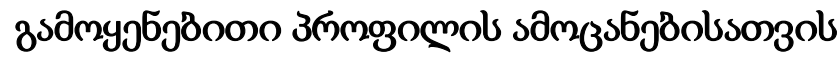

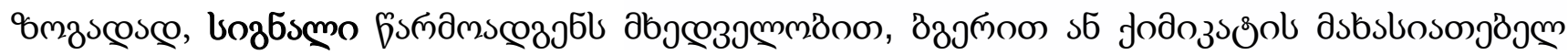

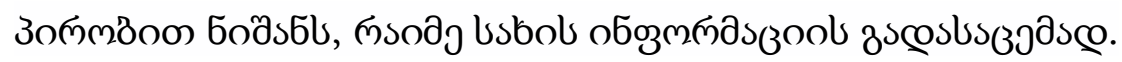

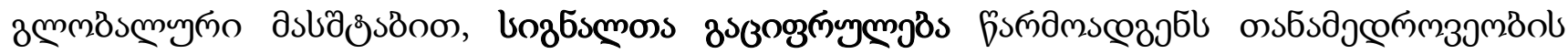

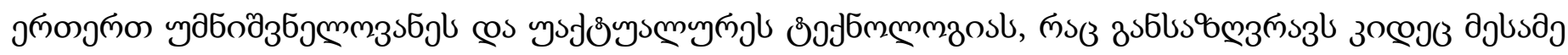

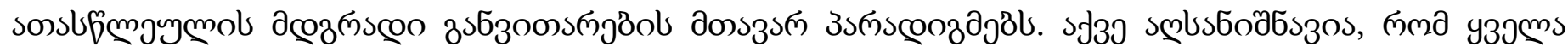

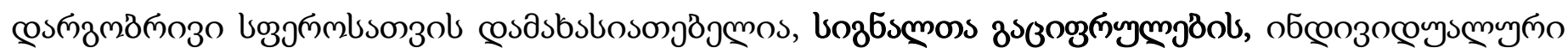

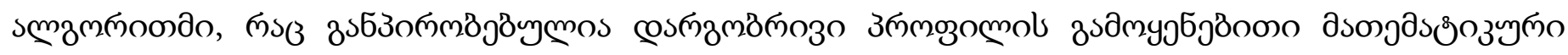

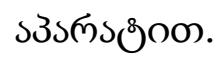

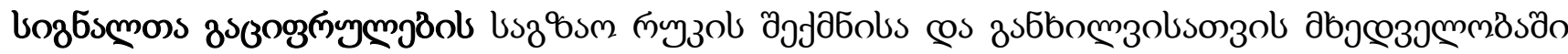

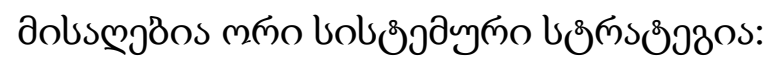

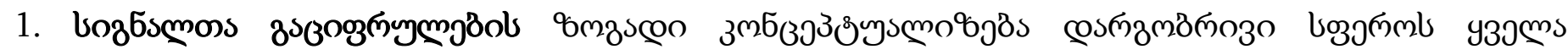
асоэбамоlsonzol.

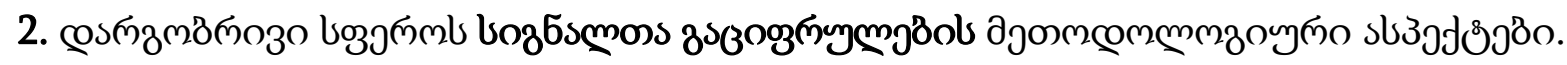




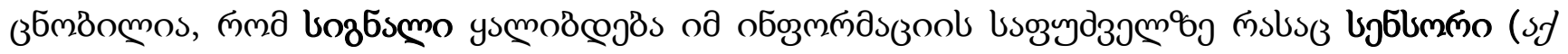

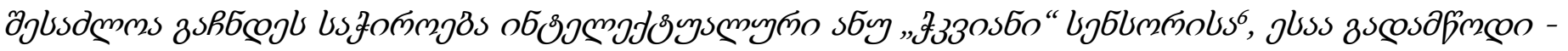

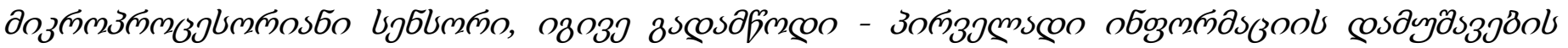

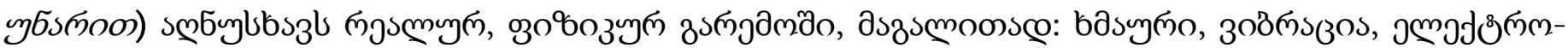

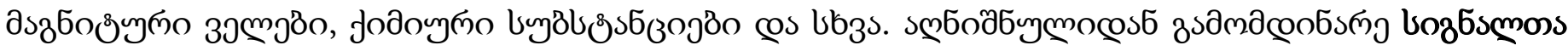

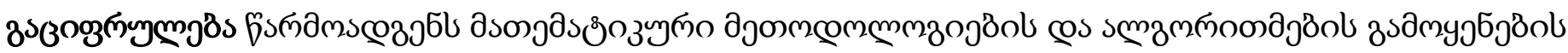

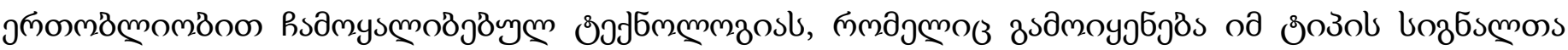

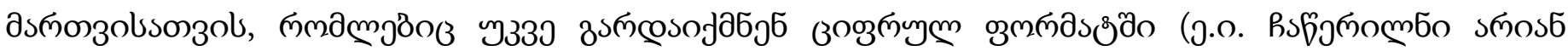

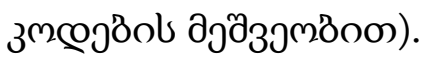

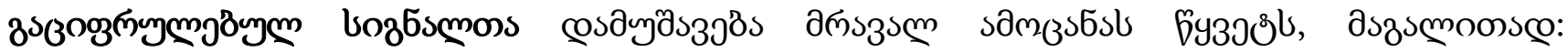

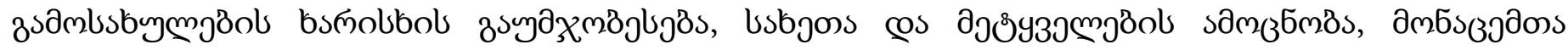

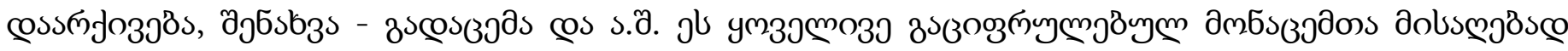

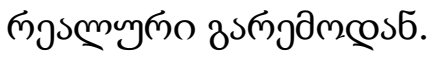

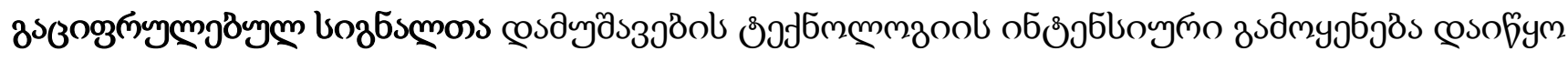

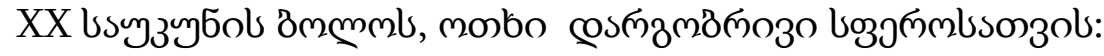

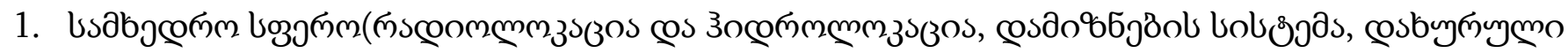
зЈздопдздаудммдs);

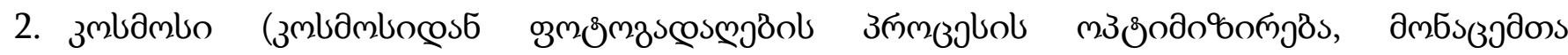

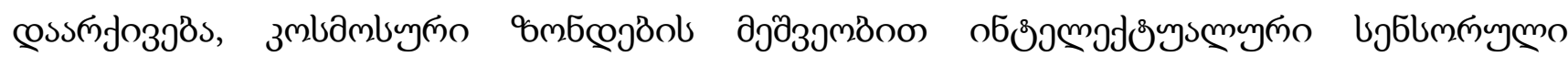
sбsmo\%o);

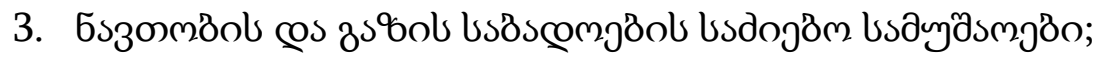

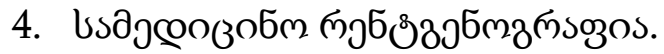

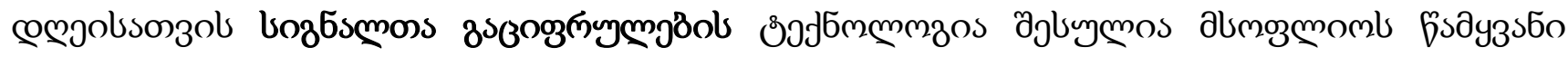

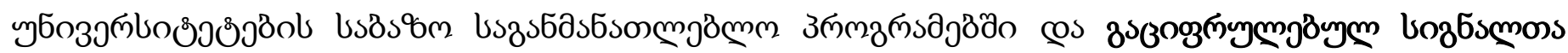

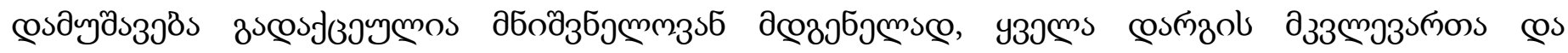

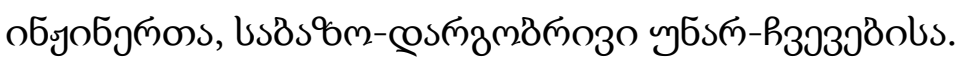

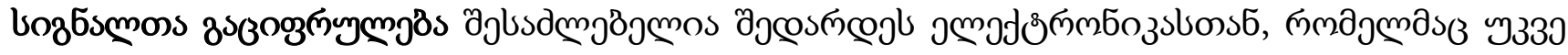

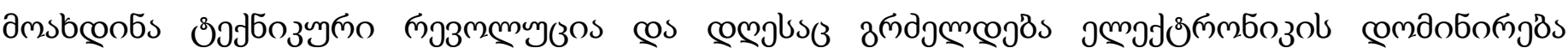

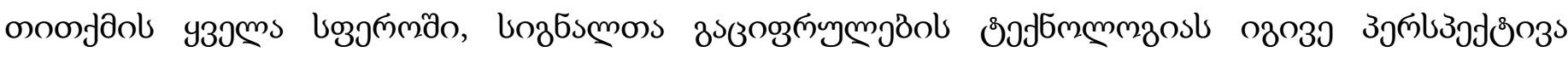

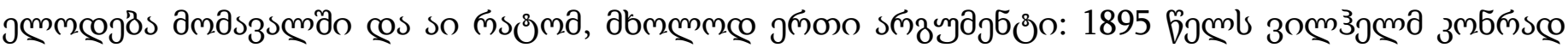

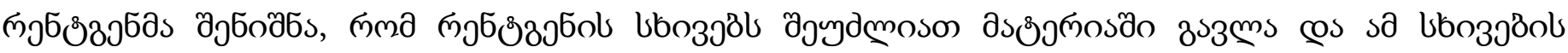

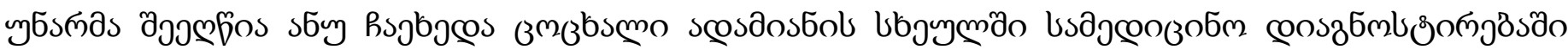

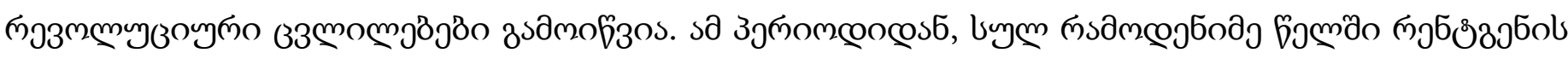

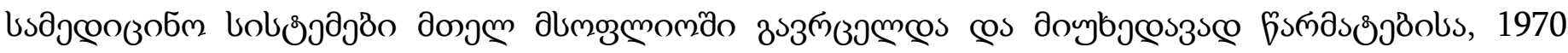

\footnotetext{
${ }^{6}$ objmolingrse - ,smart sensor"
} 


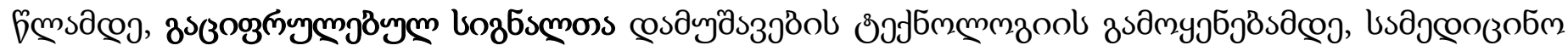
пృбฮீ

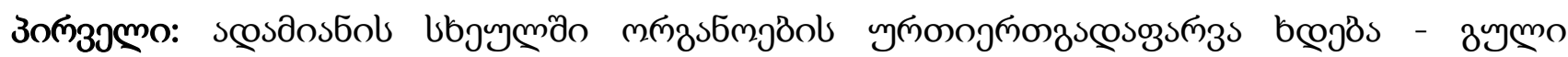

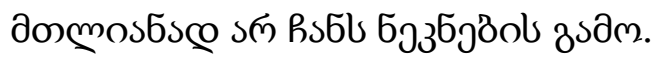

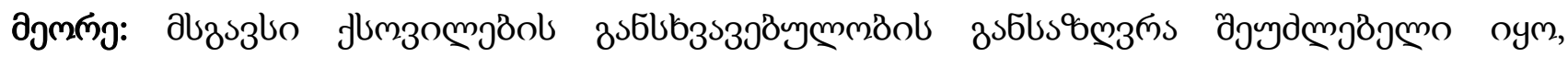

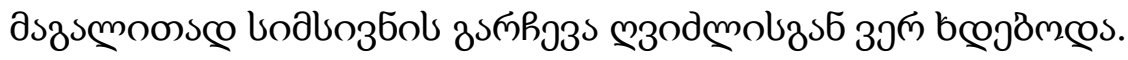

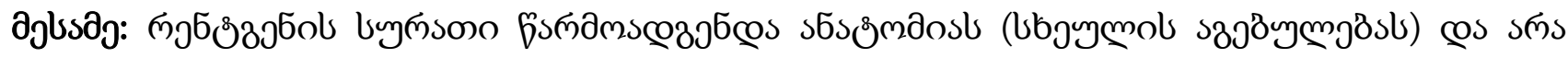

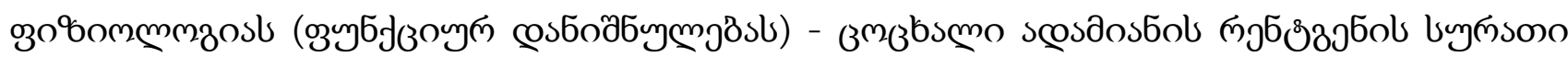

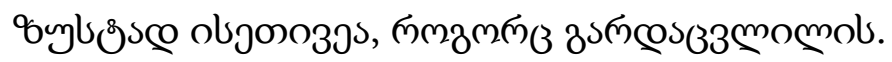

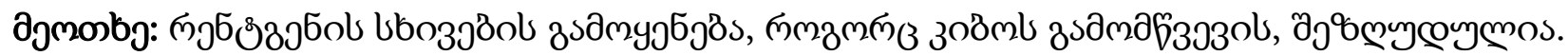

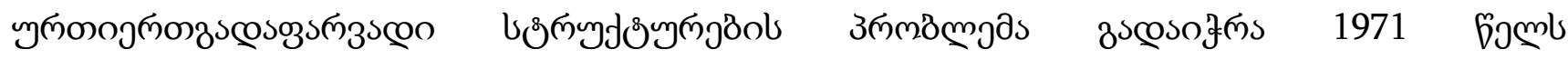

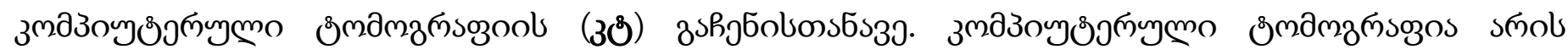

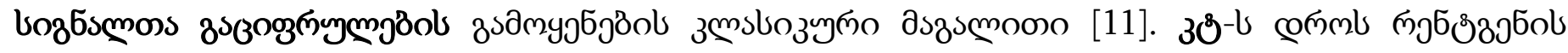

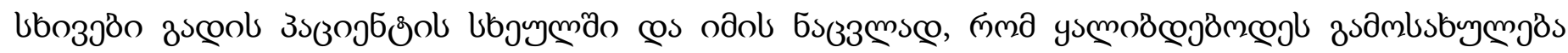

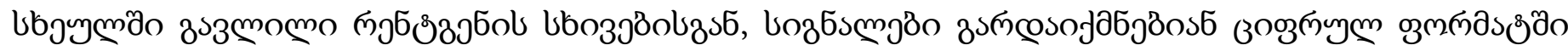

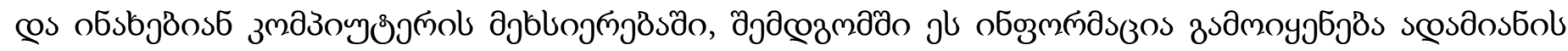

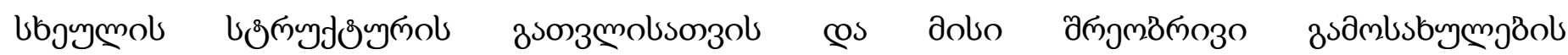

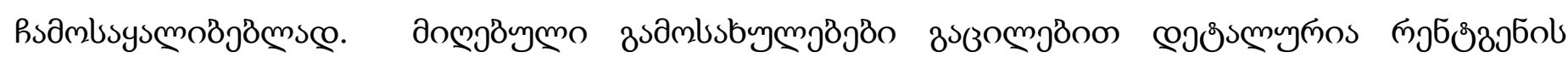

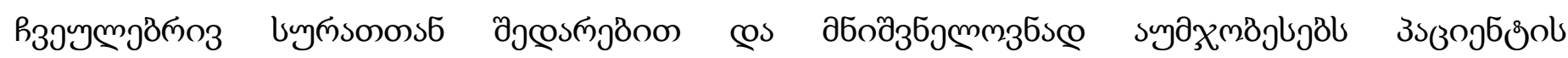

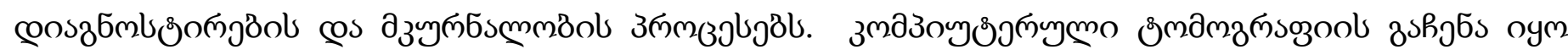

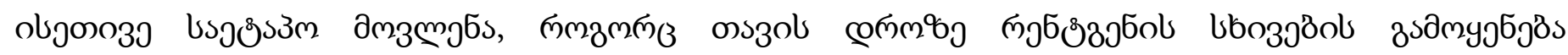

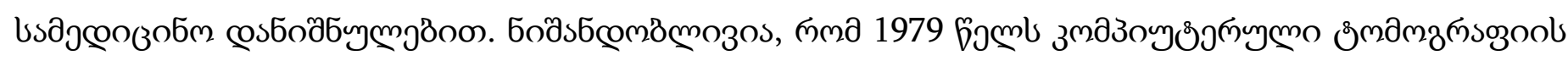

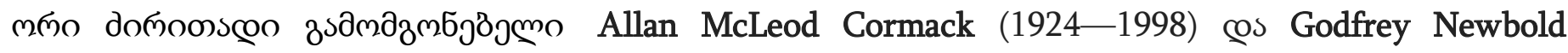

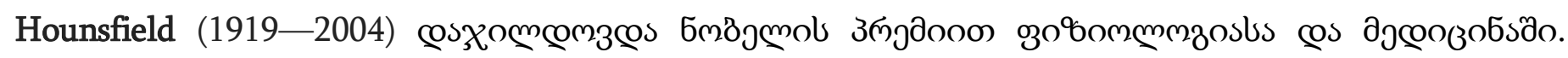

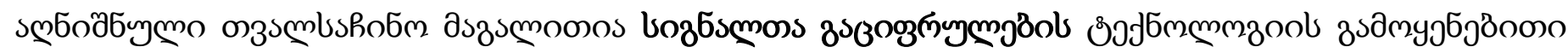

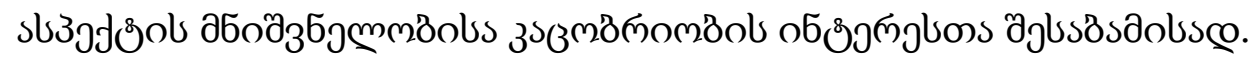

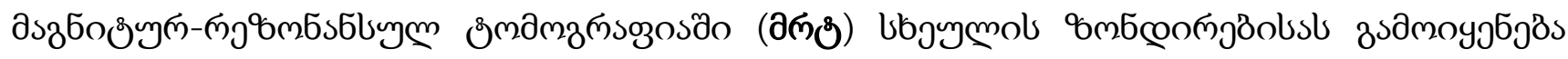

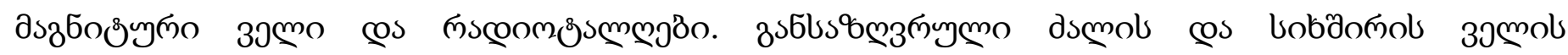

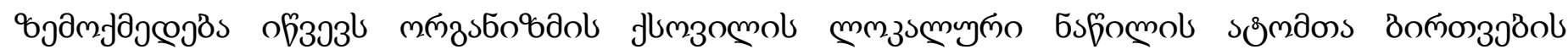

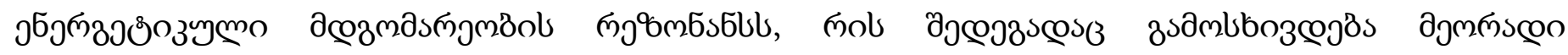

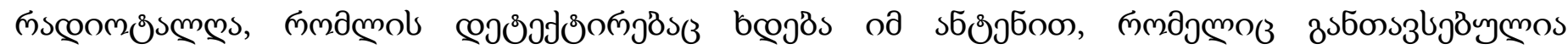

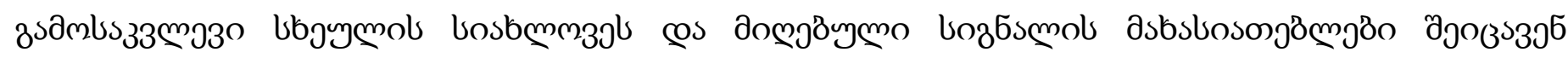

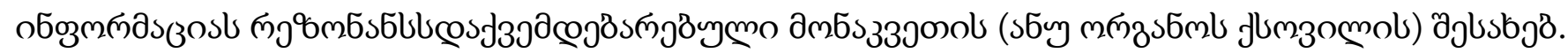

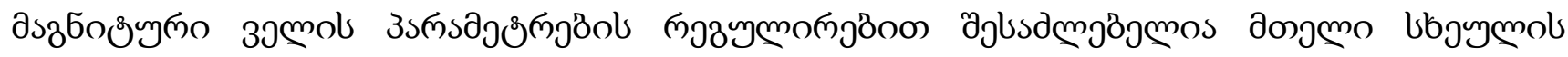

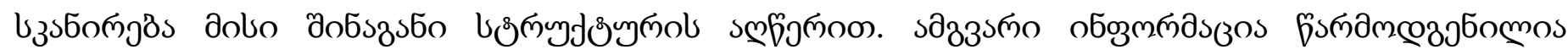




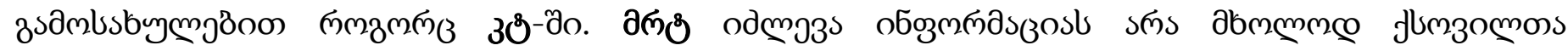

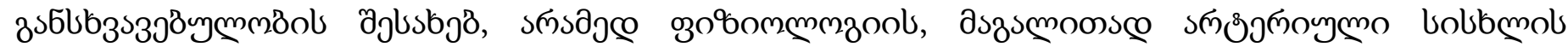

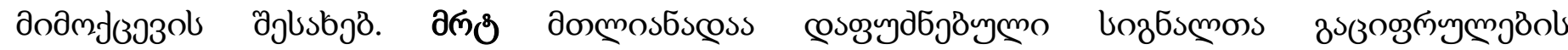

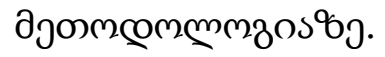

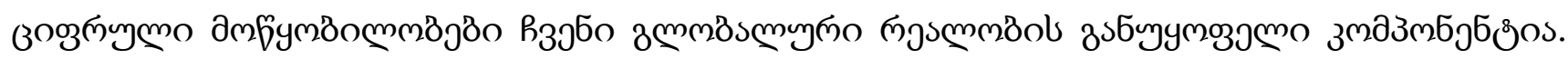

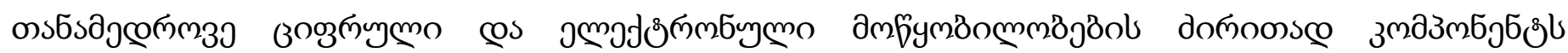

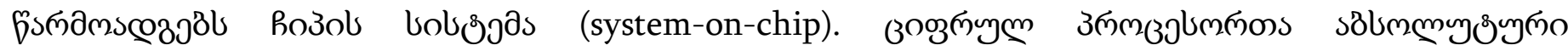

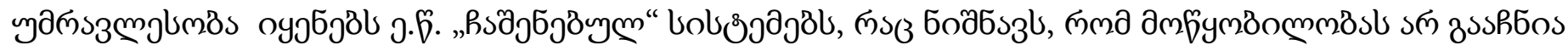

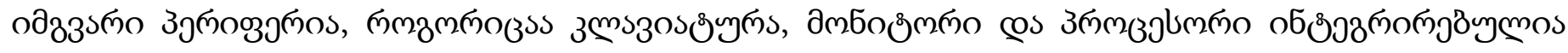

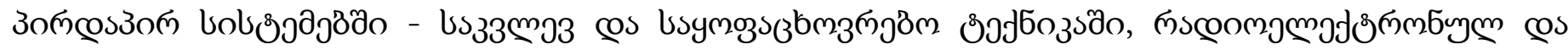

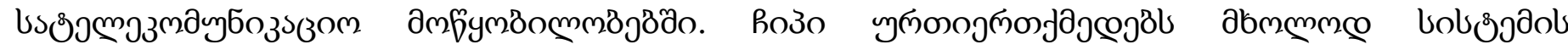

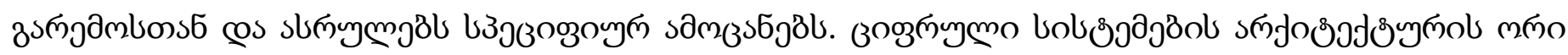

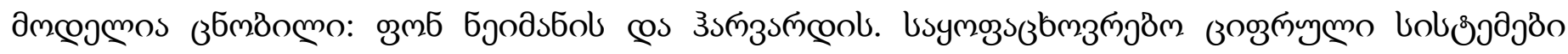

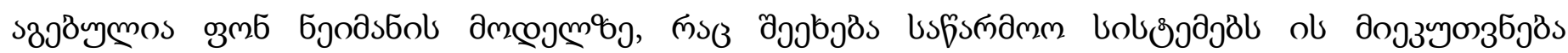

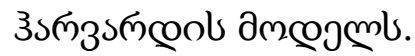

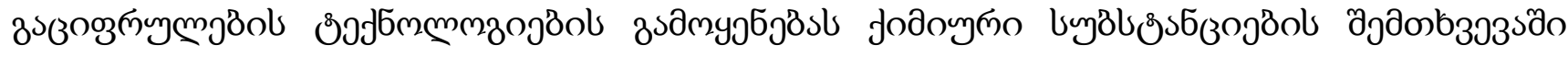

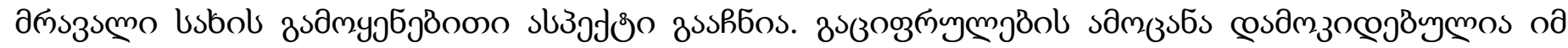

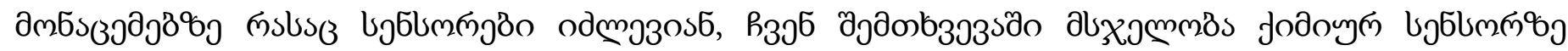

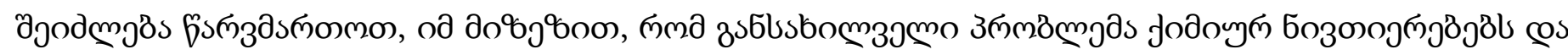

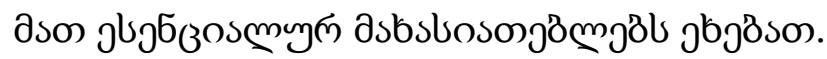

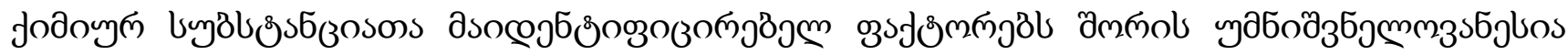

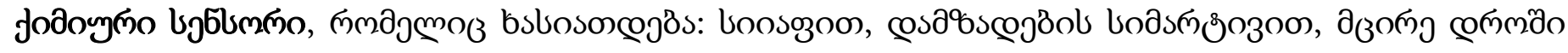

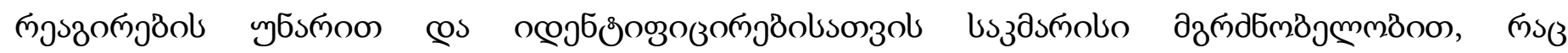

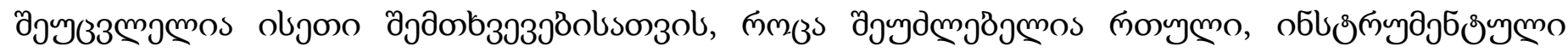
дуомщ

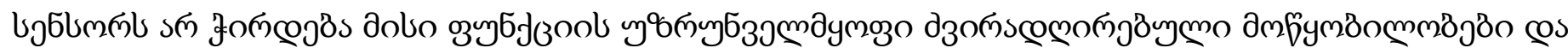
дsм\%

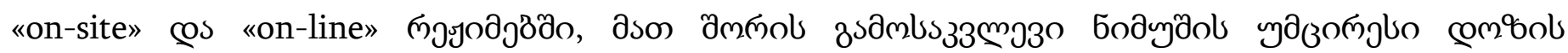

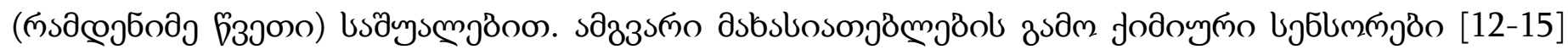

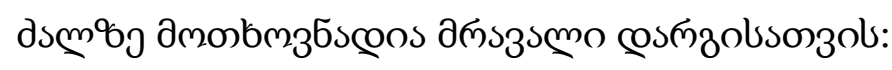

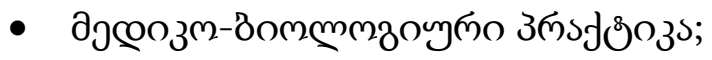

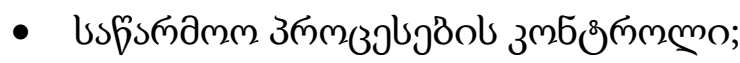

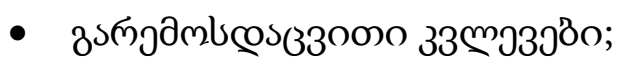

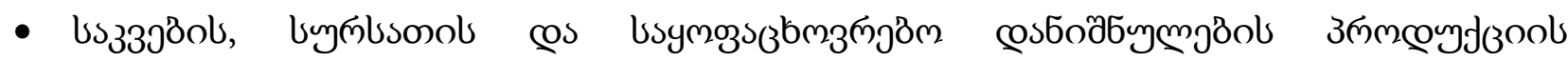

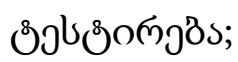

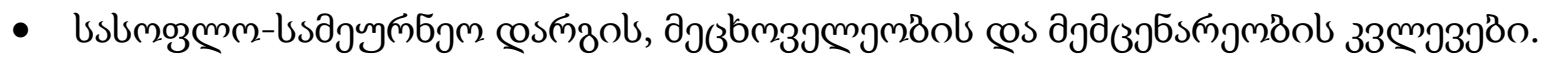




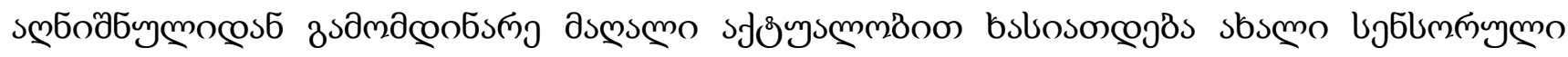

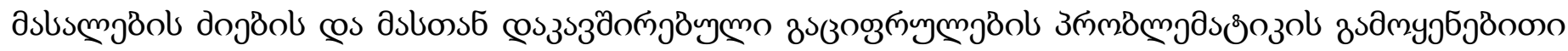

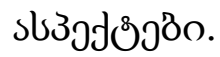

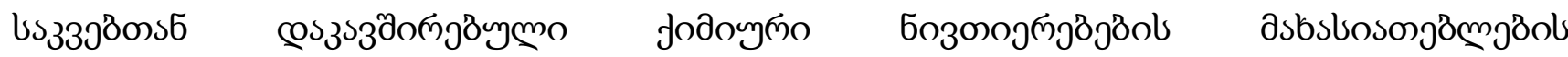

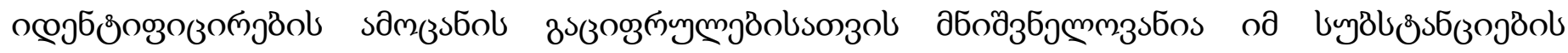

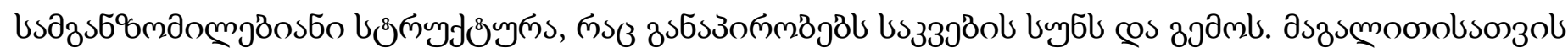

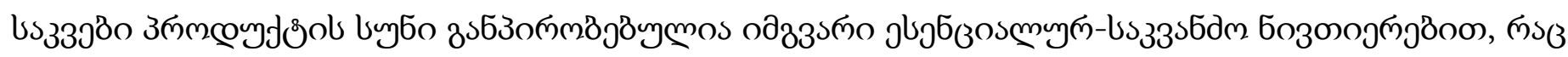

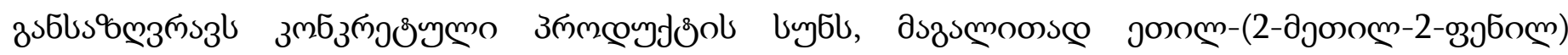

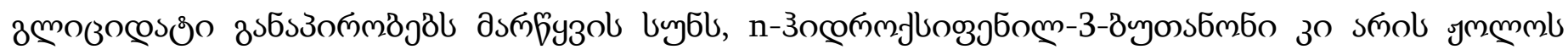

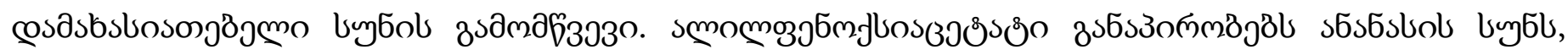

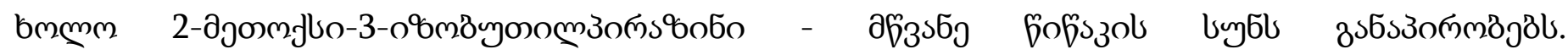

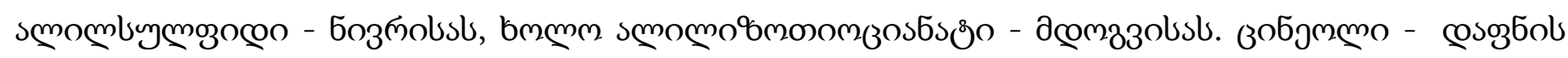

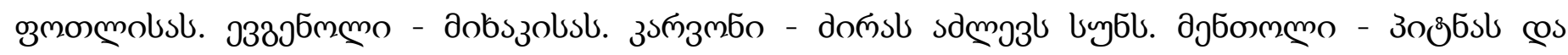

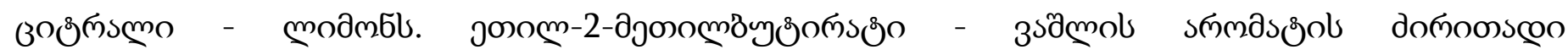

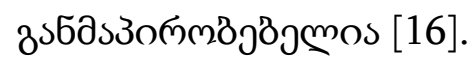

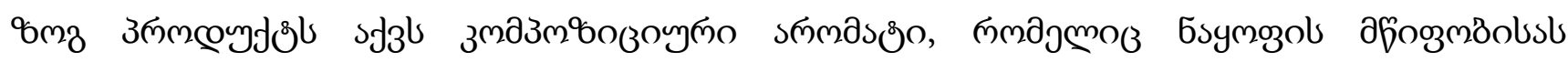

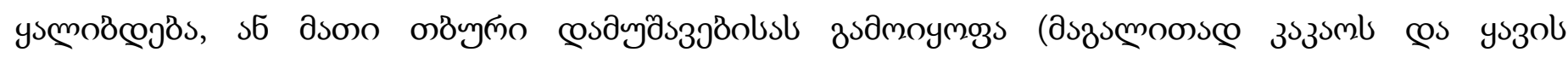

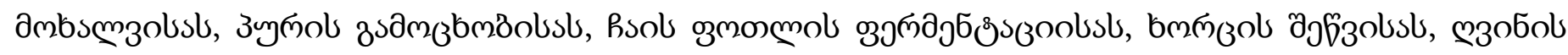

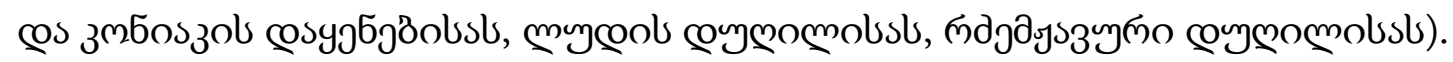

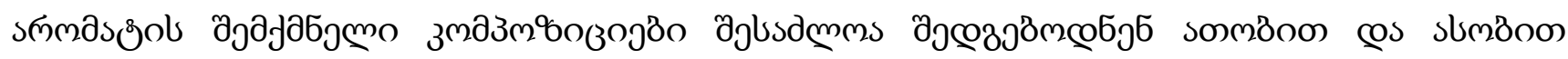

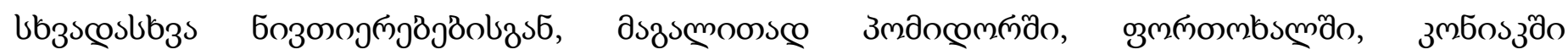

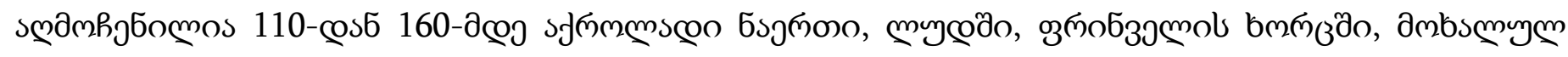

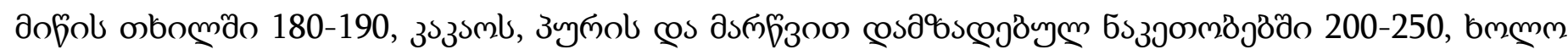

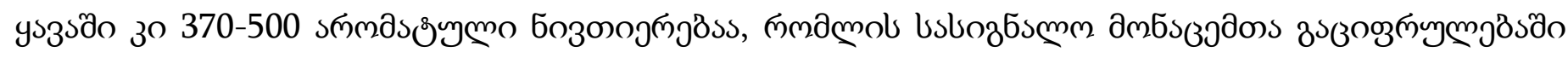

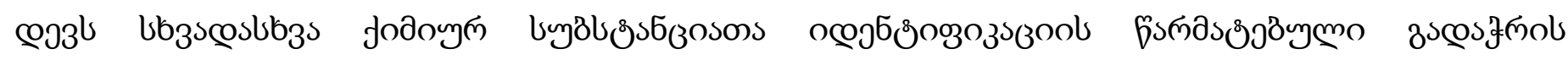

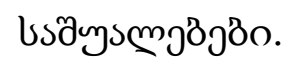

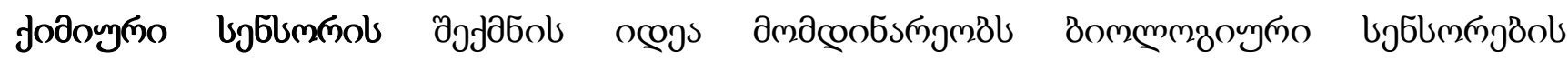

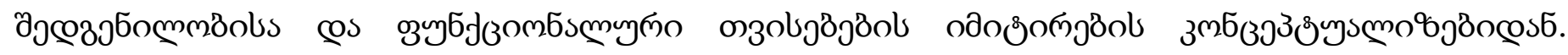

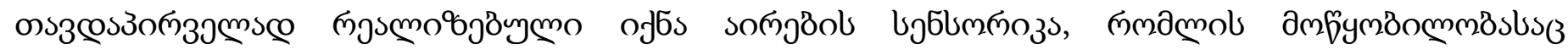

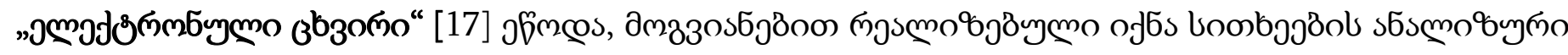

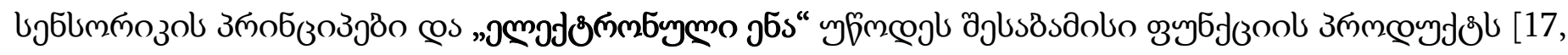
18]. 


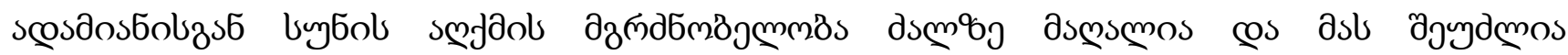

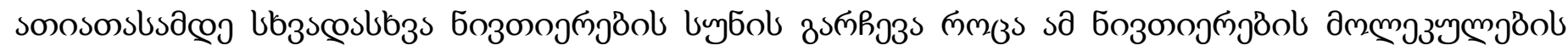

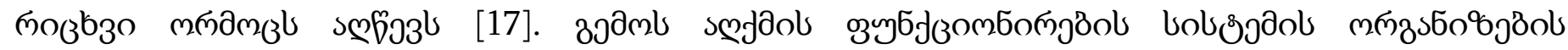

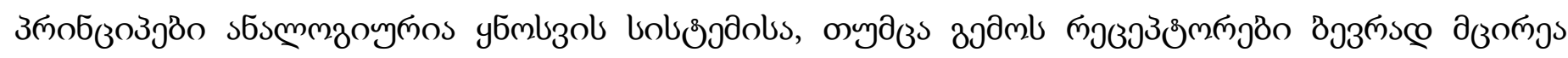

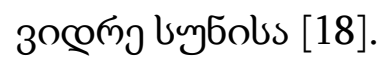

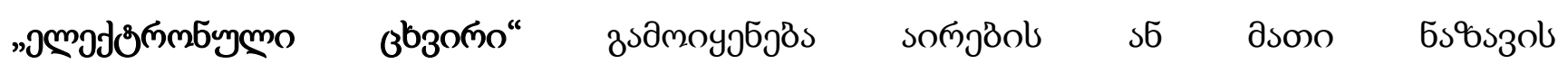

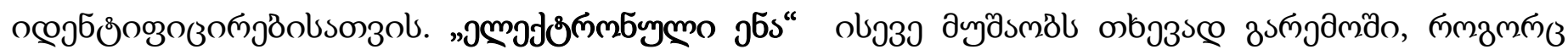

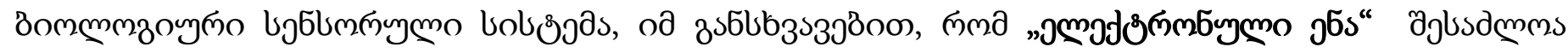

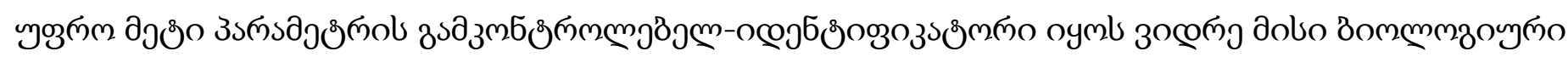

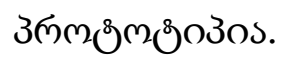

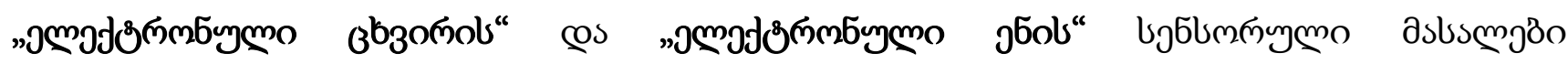

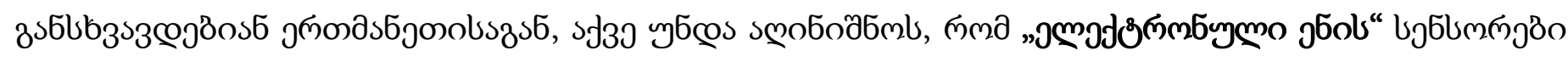

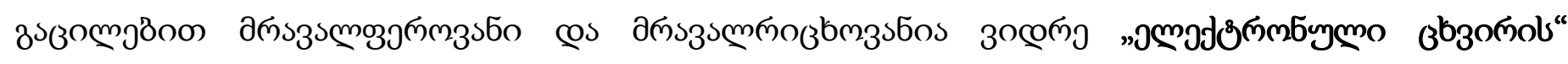

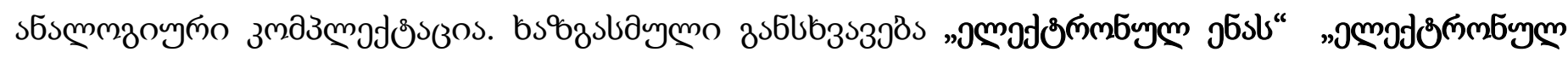

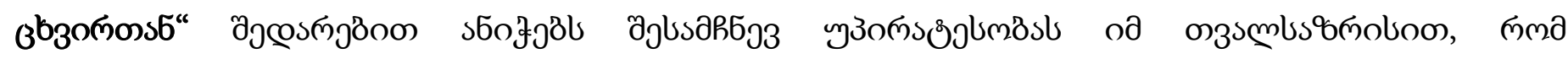

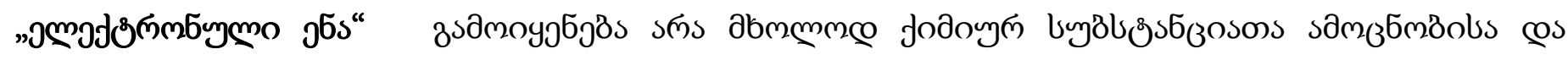

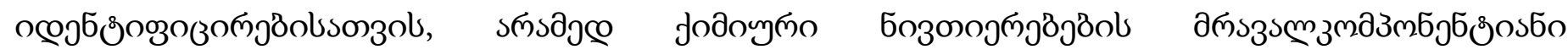

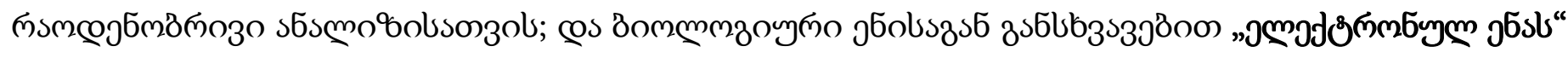

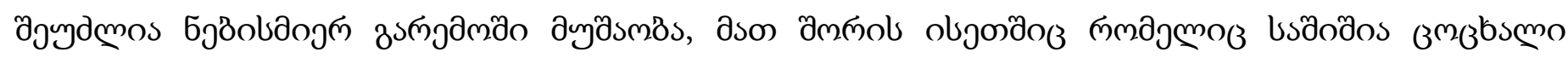

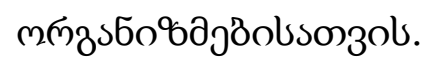

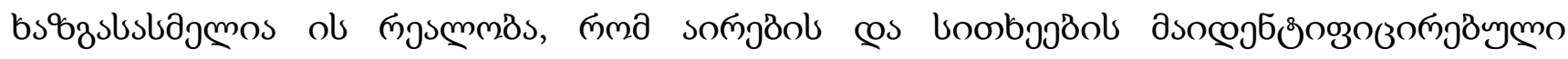

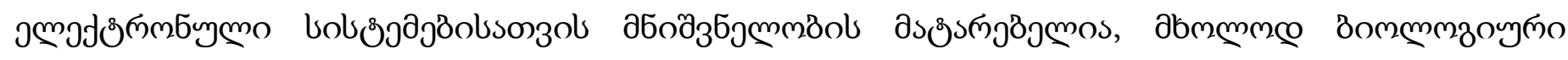

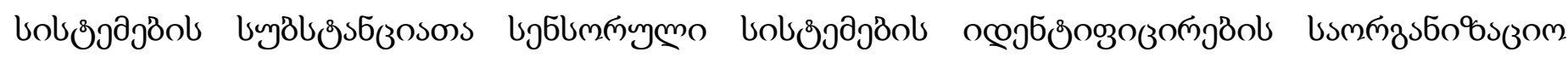

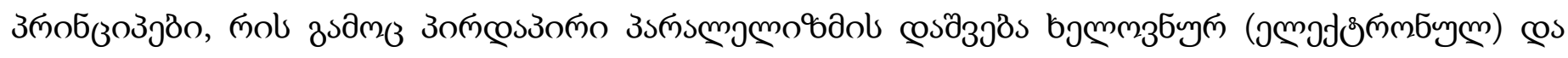

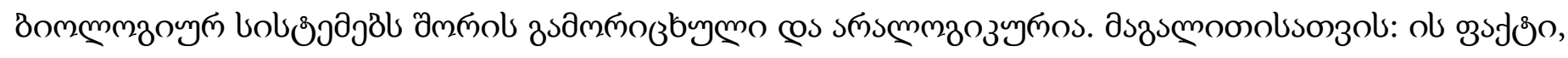

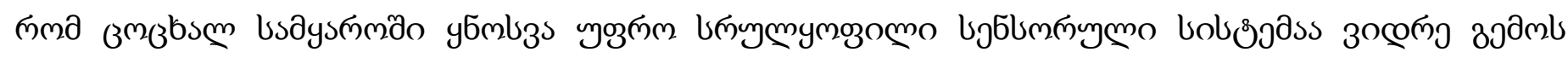

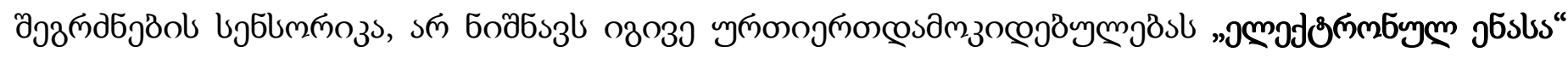

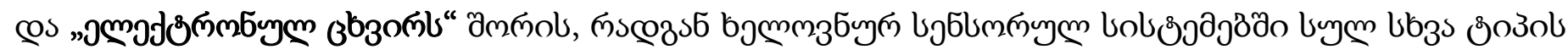

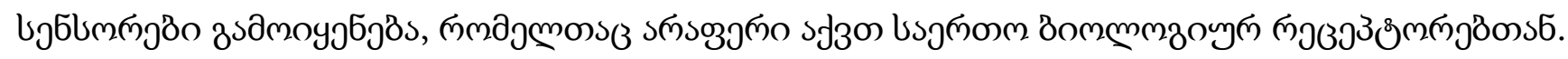

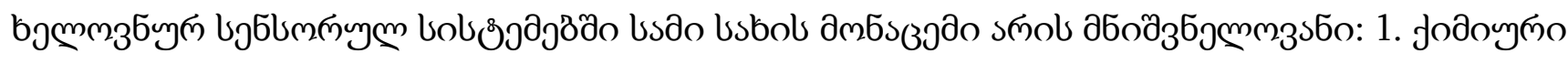

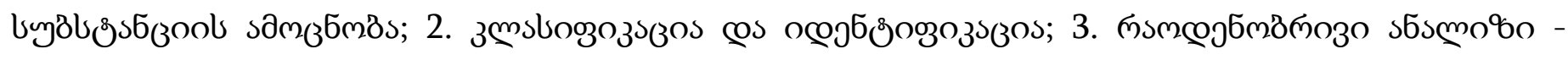

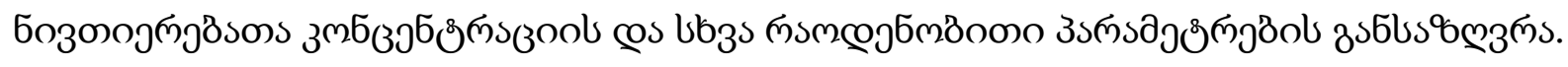

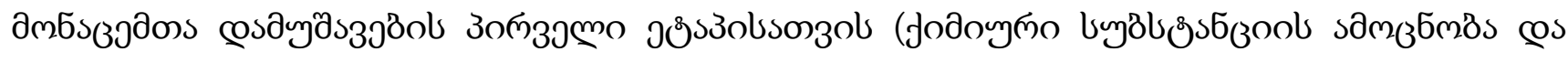

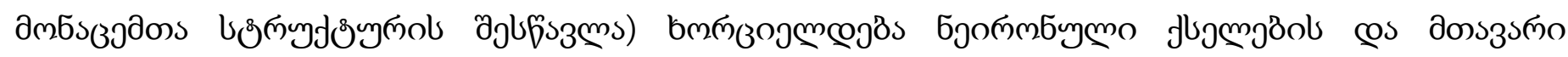




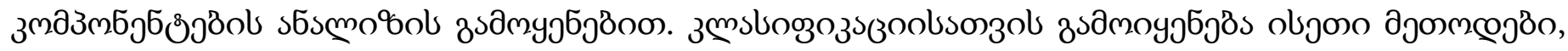

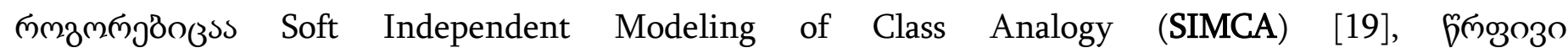

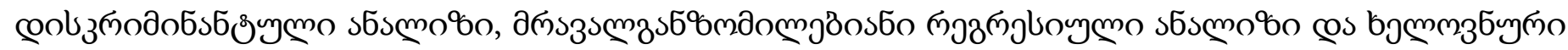

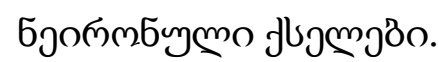

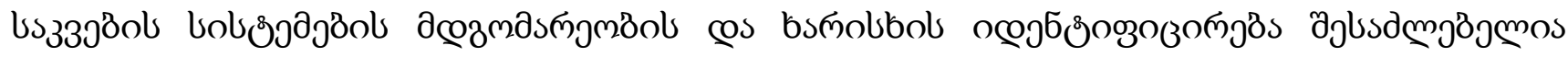

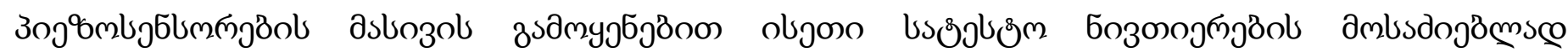

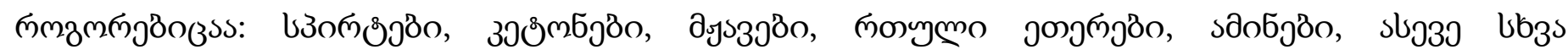

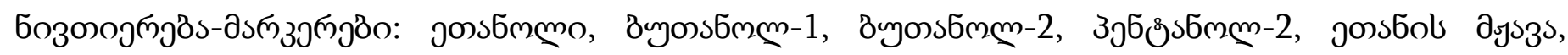

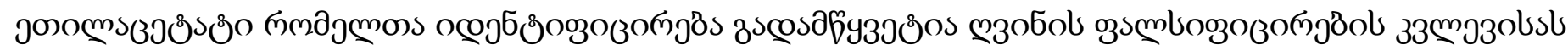
$[20]$.

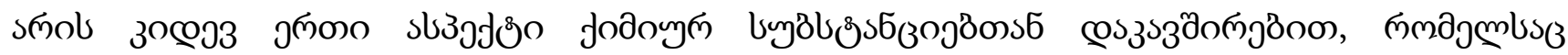

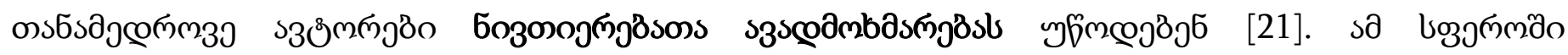

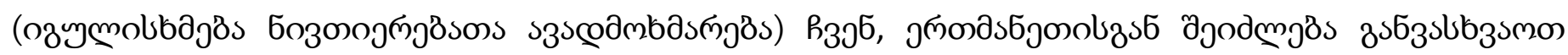

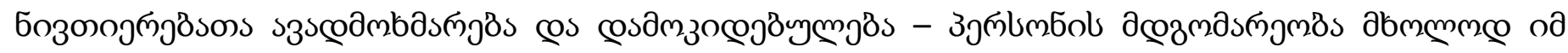

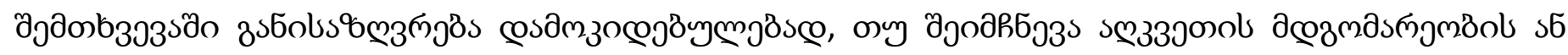

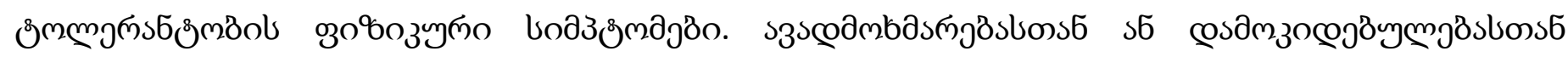

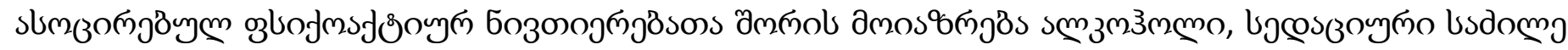

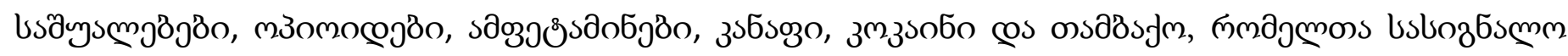

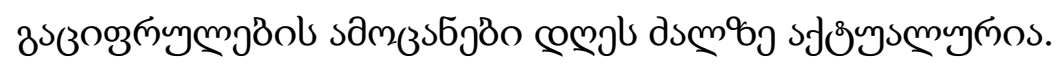

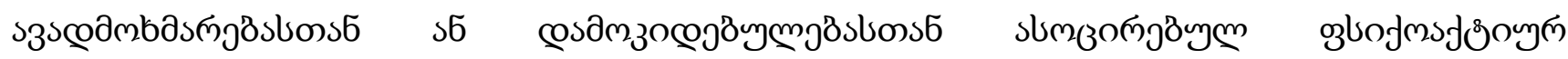

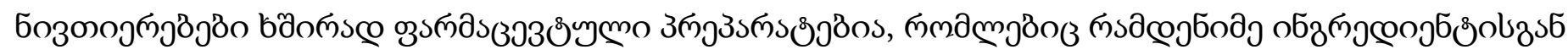

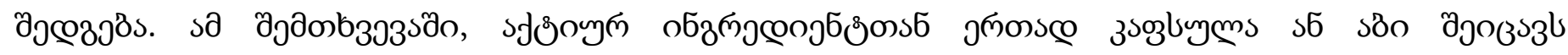

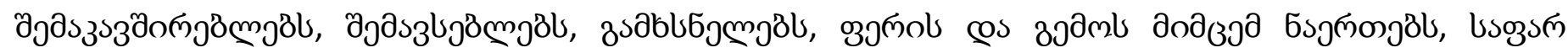

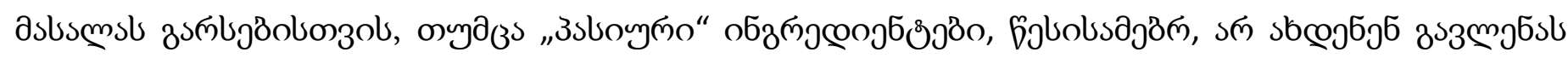

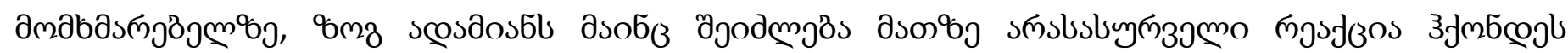

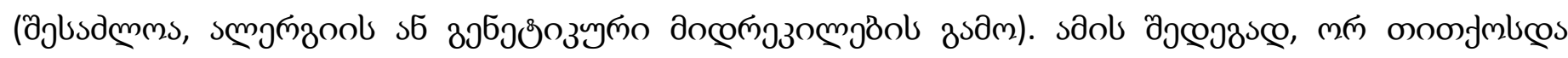

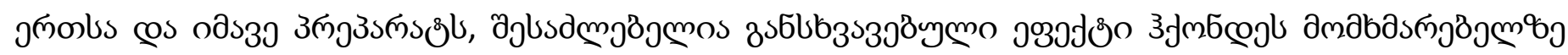

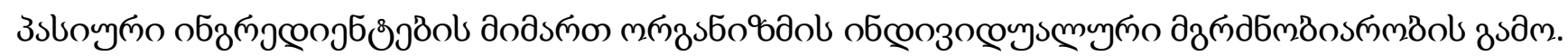

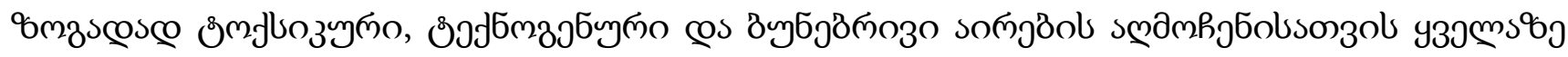

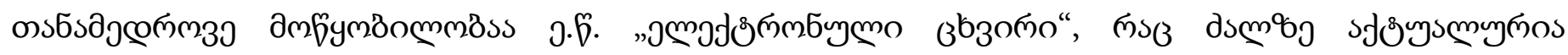

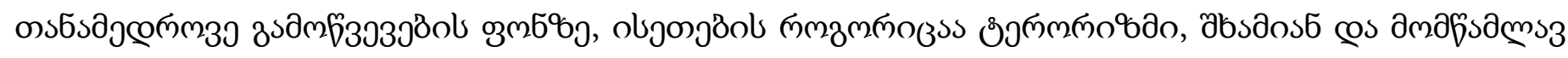

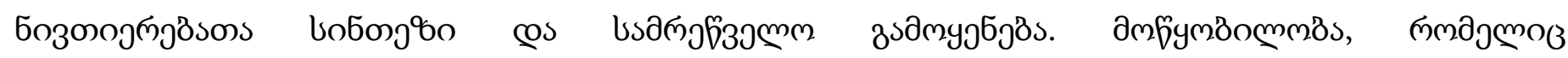

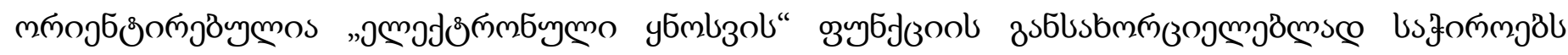

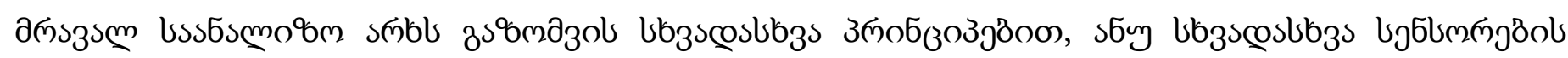

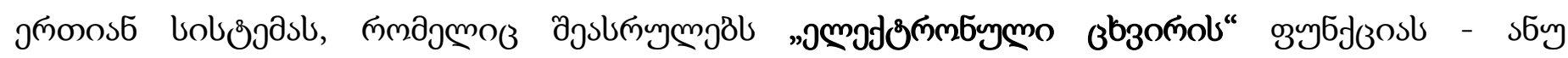

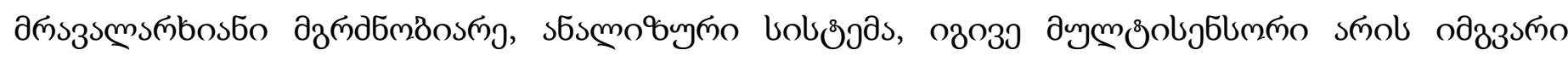




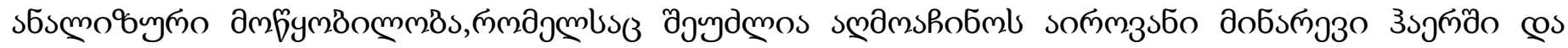

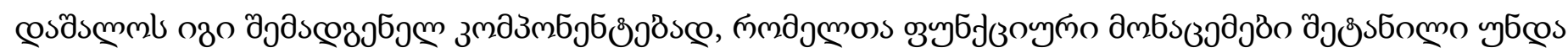

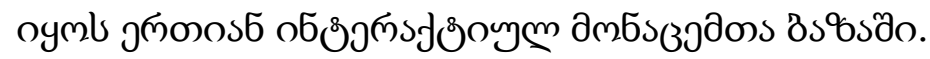

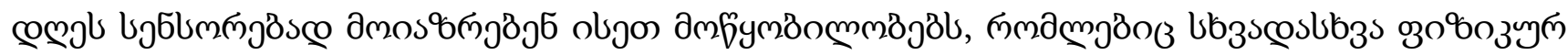

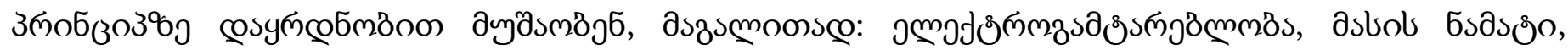

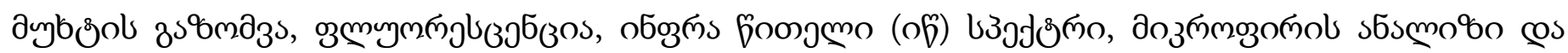

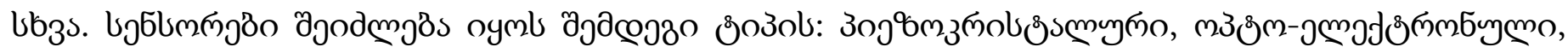

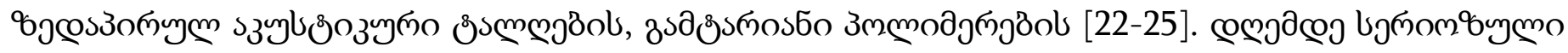

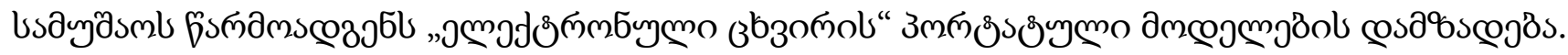

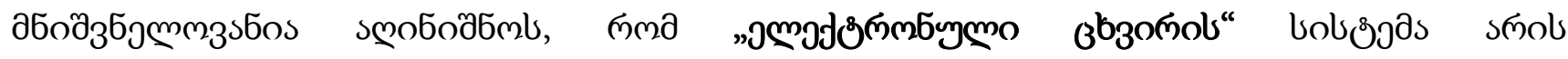

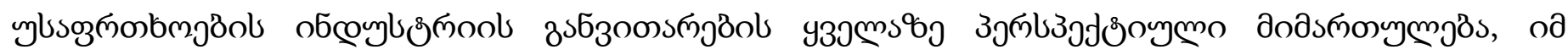

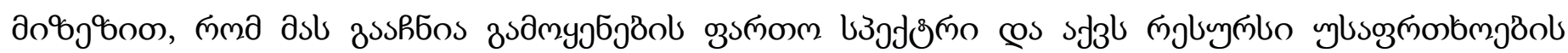

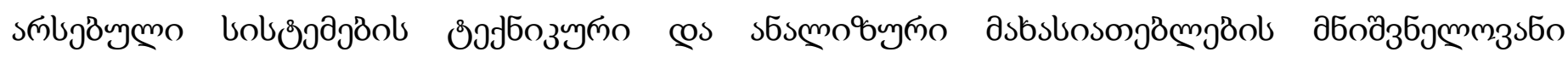

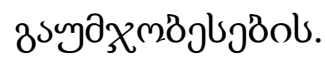

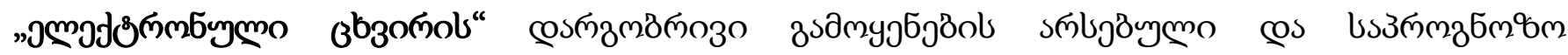

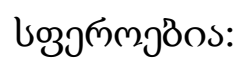

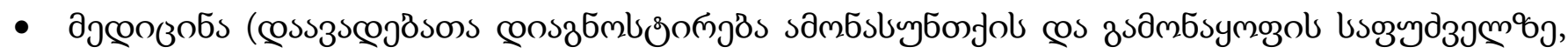

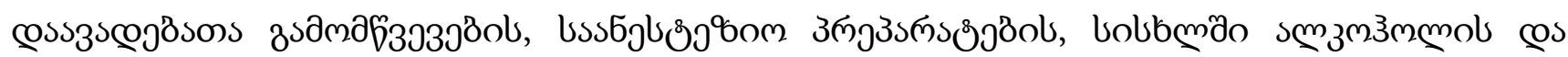

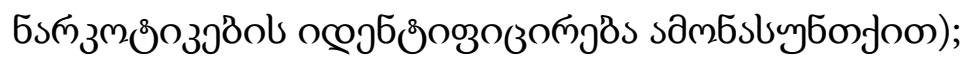

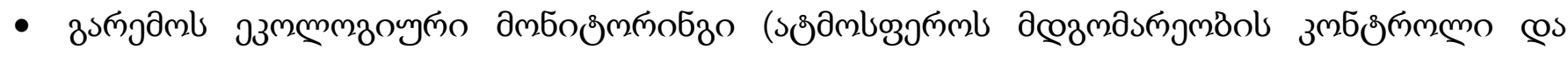

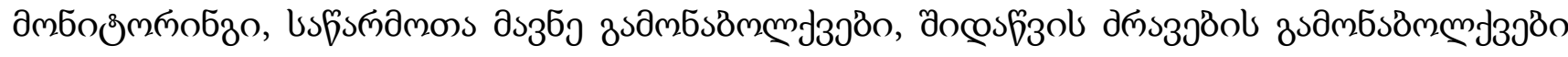
cos lbs 3 s);

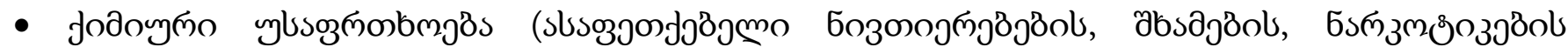

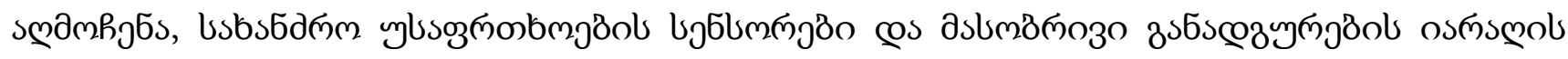

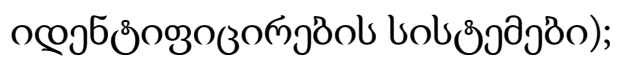

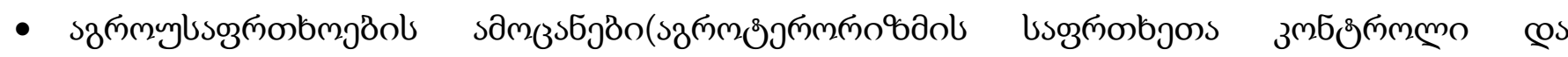

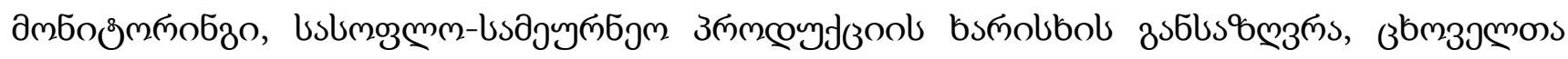

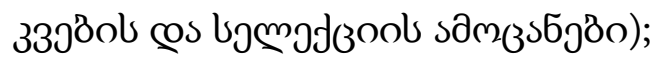

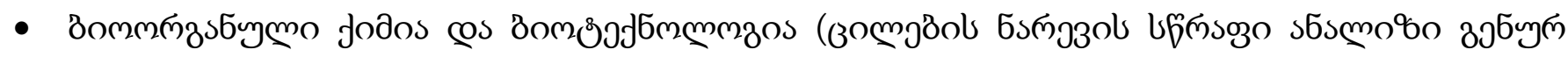

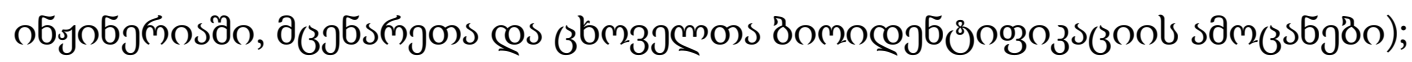

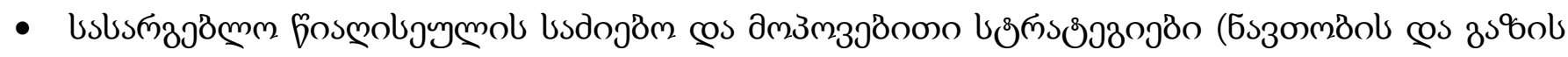

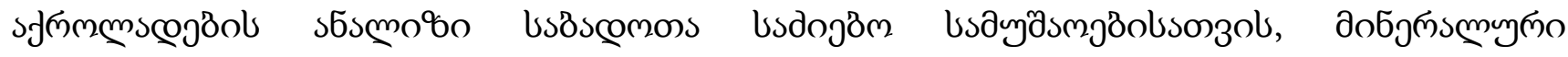

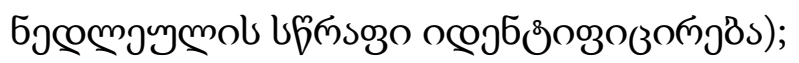

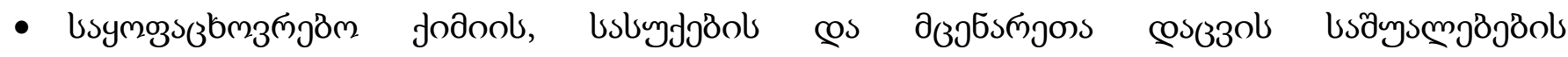

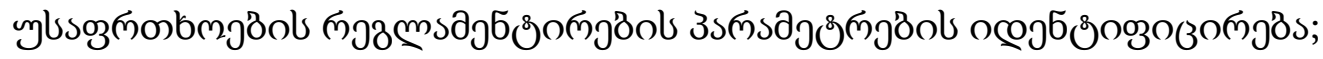




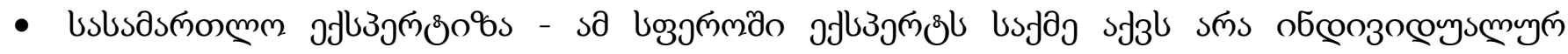

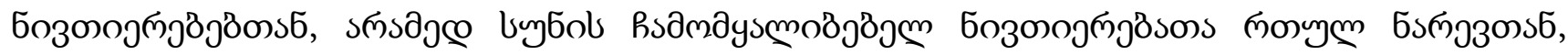

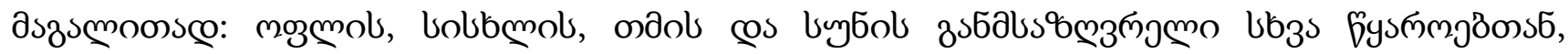

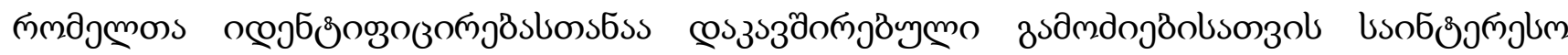

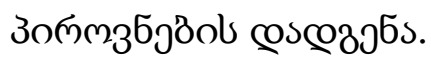

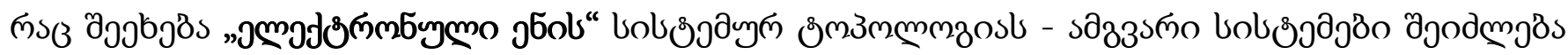

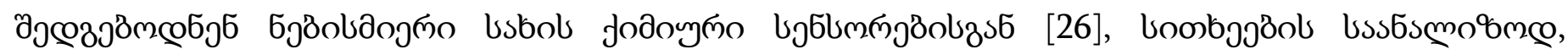

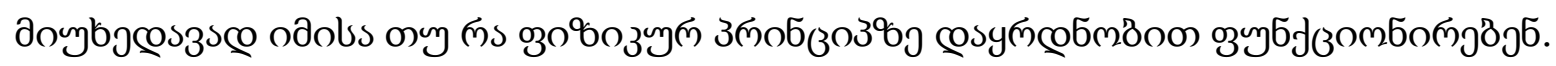

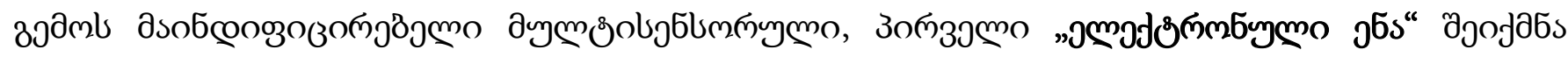

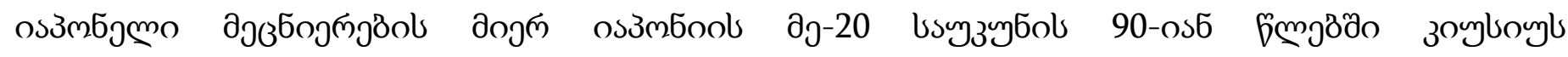

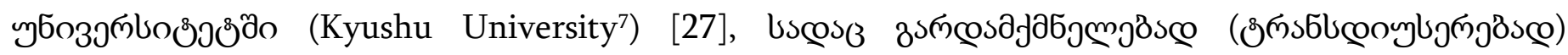

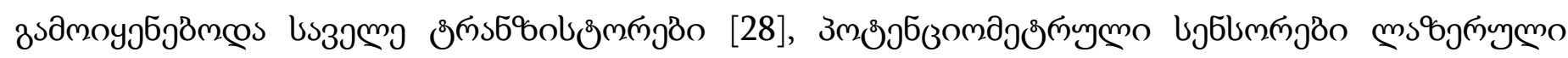

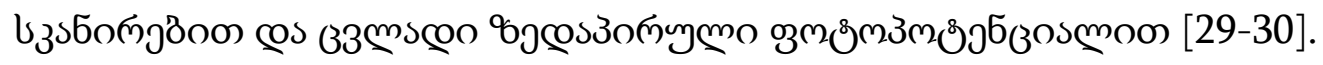

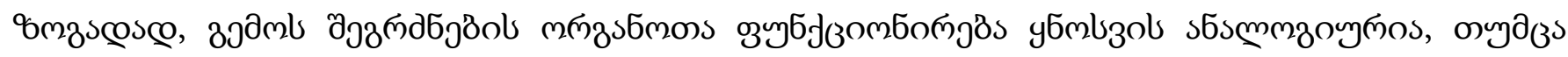

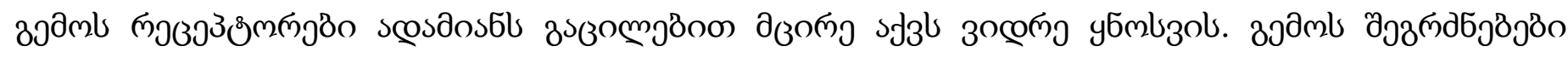

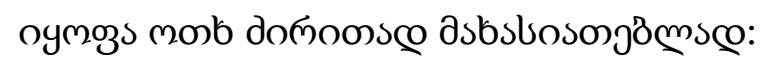

1)

2) $\partial m s \partial_{\jmath}$.

3) วэ53ว.

4) afs

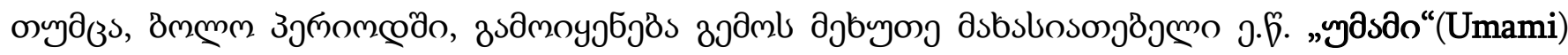

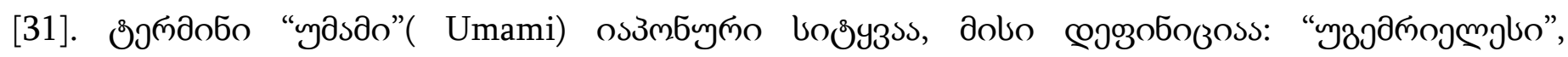

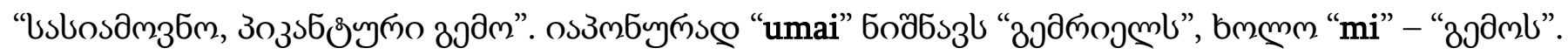

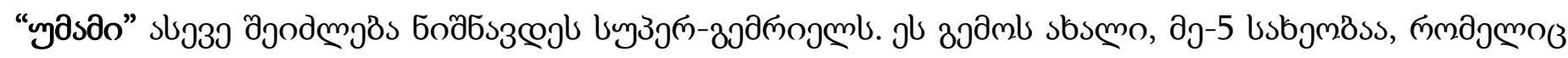

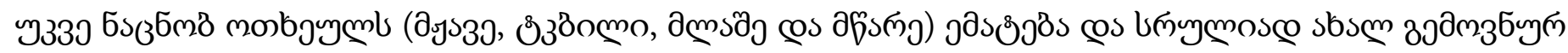

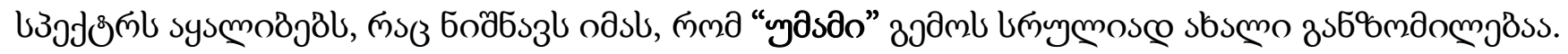

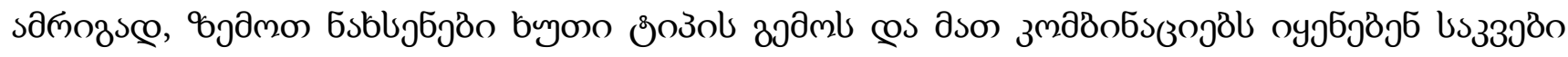
зпмкалыб

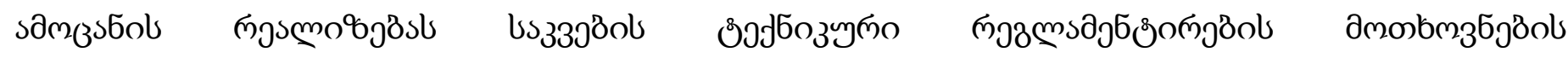

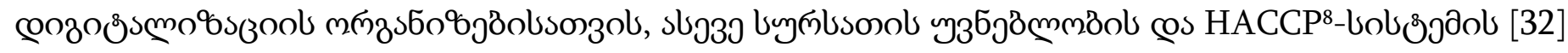

${ }^{7}$ Kyushu University - http://www.kyushu-u.ac.jp/en/university/information/

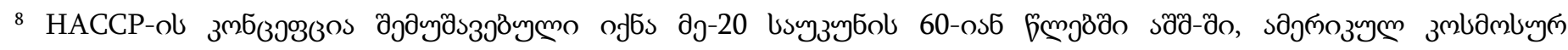

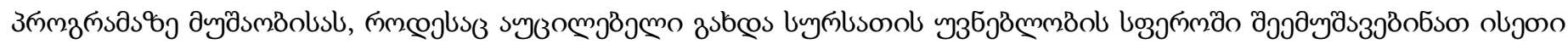




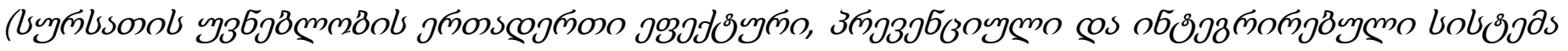

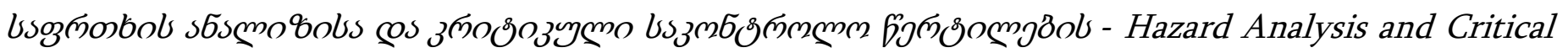

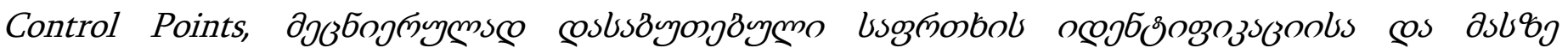

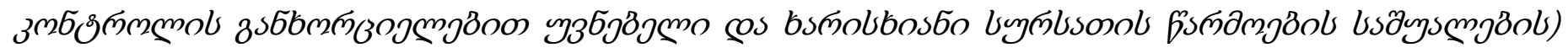

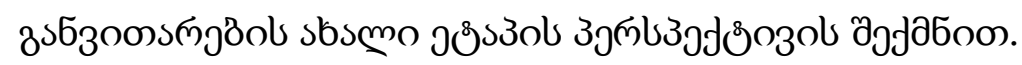

\section{cosliz36olsonzols}

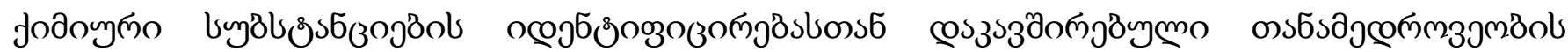

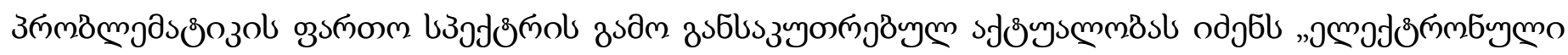

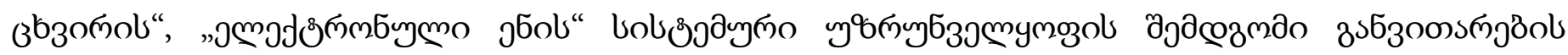

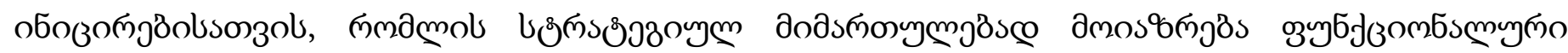

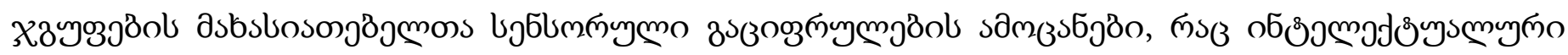

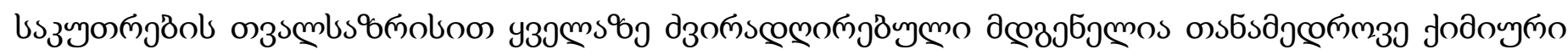

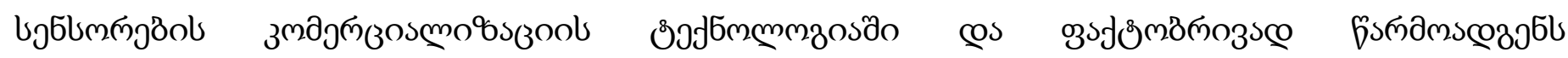

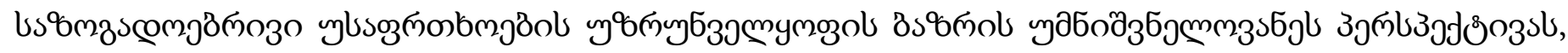

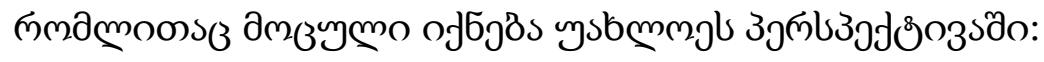

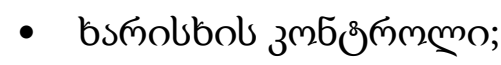

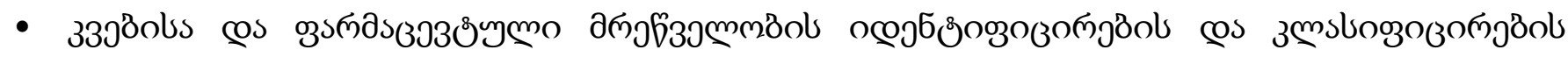
sамззsбјбо;

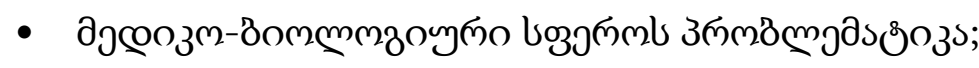

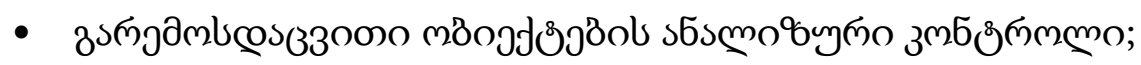

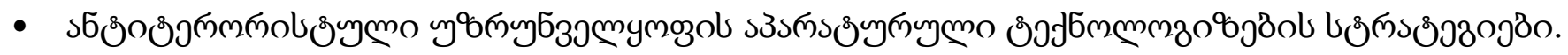

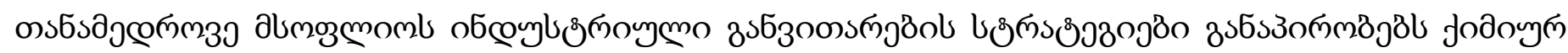

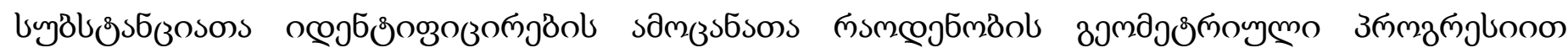

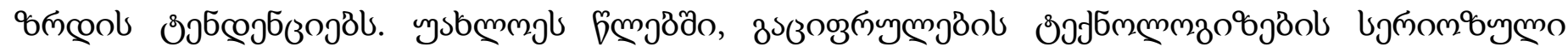

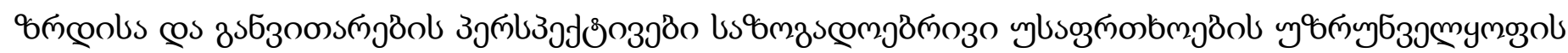

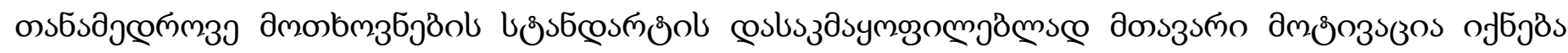

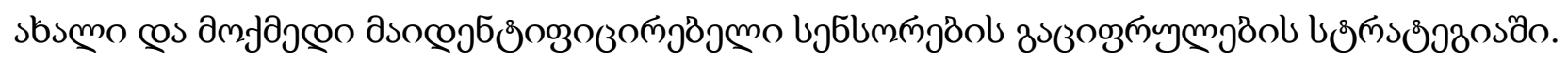

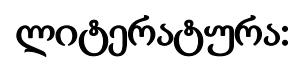

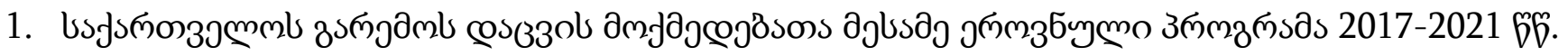
http://moe.gov.ge/res/images/file-manager/strategiuli-dokumentebi/strategiebi-gegmebi/2017-2021.pdf

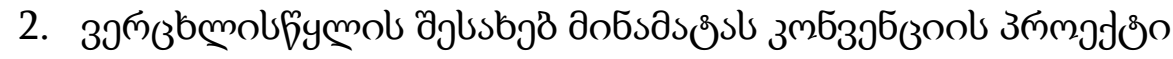

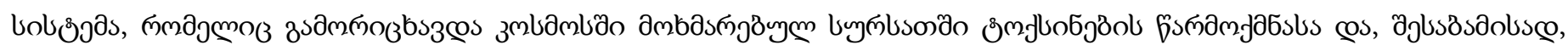

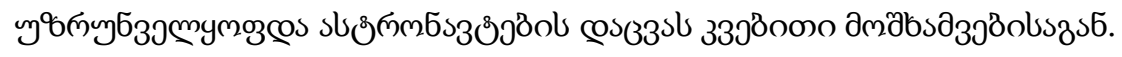




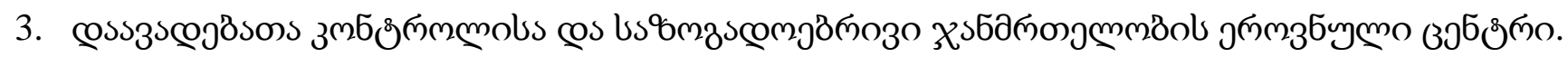

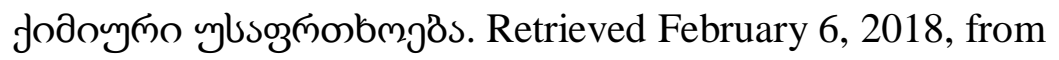
https://www.ncdc.ge/\#/pages/content/4ab48d1a-e70f-4a64-8829-794e9568eb1d

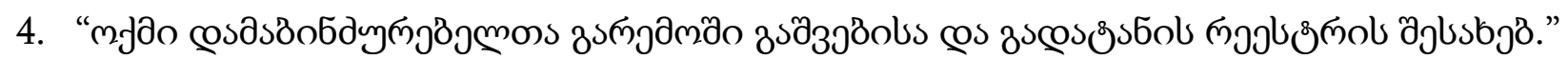

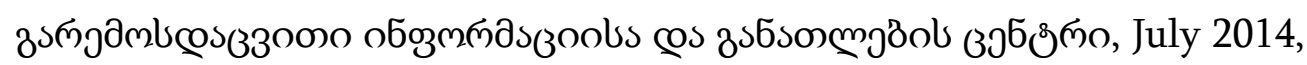
www.eiec.gov.ge/\%E1\%83\%97\%E1\%83\%94\%E1\%83\%9B\%E1\%83\%94\%E1\%83\%91\%E1\%83\%98/ Waste/Legislation/Convention/2-(299)\%E1\%83\%9D\%E1\%83\%A5\%E1\%83\%9B\%E1\%83\%98\%E1\%83\%93\%E1\%83\%90\%E1\%83\%9B\%E1\%83\%90\%E1\%83\%91\%E1\%83\%98\%E1\%83\%9C\%E1\% 83\%AB\%E1\%83\%A3\%E1\%83\%A0\%E1\%83\%94\%E1\%83\%91\%E1\%83\%94\%E1\%83\%9A\%E1\%83\% 97\%E1\%83\%90-

\%E1\%83\%92\%E1\%83\%90\%E1\%83\%A0\%E1\%83\%94\%E1\%83\%9B\%E1\%83\%9D\%E1\%83\%A8\%E1 $\% 83 \% 98-$

\%E1\%83\%92\%E1\%83\%90\%E1\%83\%A8\%E1\%83\%95\%E1\%83\%94\%E1\%83\%91\%E1\%83\%98\%E1\% 83\%A1\%E1\%83\%90-\%E1\%83\%93\%E1\%83\%90.aspx.

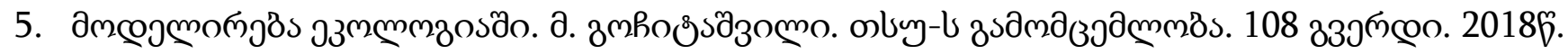

6. Investigation of integral indices of adaptation level of the human organism. O.N. Miroshnikova, E.G. Miroshnikov (A.V.Zhirmunsky Institute of Marine Biology, FEB RAS, Vladivostok). Bulletin of the Far Eastern Branch of the Russian Academy of Sciences. 2010. № 4 pp. 111-116.

7. Theoretical Aspects of the Sportsmen's Biochemical Blood Indices Change as the Index of Adaptive Processes. A.B. Lopatina - Perm National Scientific Research Polytechnic University. Pedagogicalpsychological and biomedical problems of physical culture and sports, №2 (31) 2014. Pp. 117-122.

8. Bioinformation Technology in Risk Assessment from Environmental Chemicals Influence in Human Health: Analytical Review. N. Yu. Kelina, N. V. Bezruchko, G. K. Rubtsov, O. A. Kulikova, T. Yu. Mamelina. Bulletin of TSPU. 2011. Issue 5 (107); pp. 164-169.

9. Toxic-hygienic assessment of the effect of chemical elements on human health in various ways of entering the body. Meshkov N.A. Applied toxicology, No. 1 (1), 2010, pp. 28-36.

10. The Scientist and Engineer's Guide to Digital Signal Processing. Second Edition by Steven W. Smith. California Technical Publishing, San Diego, California, 1999, pages 688.

https://users.dimi.uniud.it/ antonio.dangelo/MMS/materials/Guide to Digital Signal Process.pdf

11. G. Verrelli, L. Lvova, R. Paolesse, C. Di Natale, A. D'Amico, Metalloporphyrin-based Electronic Tongue: an application for the analysis of Italian white wines. Sensors. 2007, v. 7. pp. 2750-2762.

12. L. Tortora, M. Stefanelli, M. Mastroianni, L. Lvova, C. Di Natale, A. D’Amico, D. Filippini, I. Lundström, R. Paolesse, The hyphenated CSPT- potentiometric analytical system: An application for vegetable oil quality control. Sens. Act B.2009, v.142, pp.457-463. 
13. V. Panchuk, L. Lvova, D.Kirsanov, C. Guanais Goncalves, C. Di Natale, R. Paolesse, A. Legin, Extending electronic tongue calibration lifetime through mathematical drift correction: case study of microcystin toxicity analysis in waters. Sens. Act B. 2016, v. 237, pp. 962-968.

14. Electronic Noses and Tongues in Food Science. 1st Edition. Editors: Maria Rodriguez Mendez Series Editors: Victor Preedy. Academic Press, 2016, pages 332.

15. Food chemistry. Tutorial. For students in the specialty: Technology of production and processing of agricultural products. D.Yu. Ilyin, G.V. Ilyina. Penza 2016, 152 pages.

16. Handbook of Machine Olfaction. Electronic Nose Technology. Eds. T.C. Pearce, S.S. Schiffman, H.T. Nagle, J.W. Gardner. Darmstadt: Wiley-VCH, 2003, Ch. 1.

17. Stewart G.F., Amerine M.A. Introduction to Food Science and Technology. New York: Academic Press, 1973.

18. Tuning the significance level of SIMCA models for reducing the impact of strong class overlap: a novel approach." R. Vitale, F. Marini, C. Ruckebusch. Conference Chimiometrie. 2019.

https://chemom2019.sciencesconf.org/data/chemom2019_prixGFC_Raffaele_Vitale.pdf.

19. The information content of the gas analyzer "Electronic nose" for assessing the quality of wine. T.A. Kuchmenko, R.P. Lisitskaya, A.A. Fur coat Analytics and control 2014, v.18, No. 4, pp. 373-384.

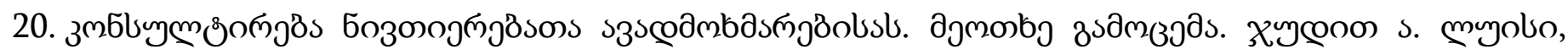

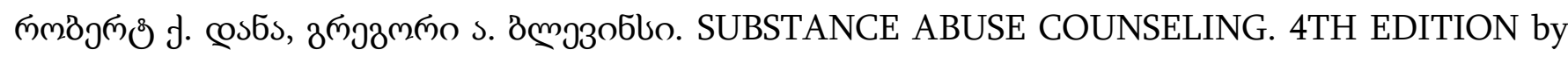
Judith A. Lewis Robert Q. Dana Gregory A. Blevins. This report is published in the frame of the project "Development of Human Resources, Evidence Base and Quality Standards in Addictology in Georgia", funded by EC, within the TEMPUS funding mechanism544219-TEMPUS-1-2013-1CZTEMPUS-SMHES (2013 - 4566 / 001 - 001). oð̊omobo 2017. 327 з3эณцо.

21. Electronic nose makes housekeeping easier. (2000, September 12). News Powered by Cision. https://news.cision.com/nordic-sensor-technologies/r/electronic-nose-makes-housekeeping-easier,e28769

22. Chemical Sensors and Chemical Sensor Systems: Fundamentals Limitations and New Trends. Andrea Orsini, Arnaldo D’Amico University of Roma “Tor Vergata” Dept. of Electronic Engineer,Via del Politecnico, 100133 Roma.

http://www.prometheus-inc.com/asi/sensors2005/papers/damico.pdf

24. Electronic Sensor Technology. https://www.estcal.com/

25. First company to market electronic noses, Alpha MOS. https://www.alpha-mos.com/about-us

26. Application of electronic nose and electronic tongue in the dairy industry. Milna Tudor Kalit, Ksenija Marković , Samir Kalit, Nada Vahčić , Jasmina Havranek. University of Zagreb, Faculty of Food Technology and Biotechnology, Department of Food Quality Control, Pierottijeva 6, 10000 Zagreb, Croatia. M. TUDOR KALIT et al.: Electronic nose and electronic tongue in the dairy industry, Mljekarstvo 64 (4), pp. 228-244, 2014.

27. Hayashi K., Yamanaka M., Toko K., Yamafuji K. Sensors and Actuators B, 1990, v. 2, pp. 205 -209. 
28. Toko K., Yasuda R., Ezaki S., Fujiyishi T. Trans. IEE Japan E, 1998, v. 118, p. 1—5.

29. Kanai Y., Shimizu M., Uchida H., Nakahara H., Zhou C.G., Maekawa H., Katsube T. Sensors and Actuators B, 1994, v. 20, № 2-3, p. 175-179.

30. Sasaki Y., Kanai Y., Uchida H., Katsube T. Ibid., 1995, v. 25, № 1-3, p. 819—822.

31. Kawamura Y., Kare M.R. Umami: A Basic Taste. New York: Marcel Dekker, 1987.

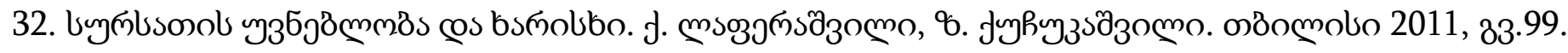




\title{
For the problem of digitalization of the chemical substances
}

\author{
M. Matsaberidze', J. Kerkadze², I. Janelidze³, G. Jandieri ${ }^{4}$ \\ ${ }^{1}$ Georgian Technical University, Doctor of Chemistry, Professor Orcid ID: https://orcid.org/0000-0002-3228-1447 \\ ${ }^{2}$ Member-Corresponding of the Georgian Engineering Academy. Georgian Technical University; Faculty of Chemical \\ Technology and Metallurgy; Department of Environmental Engineering and Ecology; Ph. Doctor, Associate Professor; \\ e-mail:j.kerkadze@gtu.ge \\ ${ }^{3}$ Georgian Technical University: Faculty of Chemical Technology and Metallurgy, PhD in Chemistry, Professor (Assistant) \\ E-mail: i.janelidze@gtu.ge; Orcid ID: https://orcid.org/0000-0002-9961-7638 \\ ${ }^{4}$ Metallurgical Engineering and Consulting Ltd; Director, PhD (Technical Sciences), \\ E-mail: gigo.jandieri@gmail.com; Orcid ID: https://orcid.org/0000-0003-2976-1201
}

\begin{abstract}
Characteristics of chemical substances or functional groups can be presented with different signals. In general, the signal is called the parameter of the process we use to refresh, register and for transmission. There are lots of signal transmissions and processing example: Human sensory organs (visual, hearing sensations) signals the brains to provide information to the environment with different information and in the brain to process the signals and make decisions on the brain by the brain.

The task of processing the signal function of the chemical substance is to identify the existing data in the signal and convert them to make a decision in a convenient form that serves the determination of the chemical compound.
\end{abstract}

The signal analysis underlines not only its mathematical transformation, but also the conclusion of the conclusions of the specific processes and objects as a result of this transformation.

The goal of the signal analysis can be:

1. Determine the numerical parameters of the signal;

2. Dispersal of the signal as an elemental parameter to compare the different signal characteristics;

3. Quantification and assessment of dependence between signals.

Keywords: Minamata convention; Biochemical demand for oxygen; Digital system; Signal intensity; Computer tomography (Ct); Magnetic resonance tomography (mrt); Chemical sensor; "Electronic nose"; "Electronic tongue". 